\title{
On nonseparating contact hypersurfaces in symplectic 4-manifolds
}

\author{
Peter Albers \\ BARNEY BRAMHAM \\ CHRIS WENDL
}

\begin{abstract}
We show that certain classes of contact 3-manifolds do not admit nonseparating contact type embeddings into any closed symplectic 4-manifold, eg this is the case for all contact manifolds that are (partially) planar or have Giroux torsion. The latter implies that manifolds with Giroux torsion do not admit contact type embeddings into any closed symplectic 4-manifold. Similarly, there are symplectic 4-manifolds that can admit smoothly embedded nonseparating hypersurfaces, but not of contact type: we observe that this is the case for all symplectic ruled surfaces.
\end{abstract}

32Q65; 57R17

\section{Introduction}

\subsection{Main results}

Let $(W, \omega)$ denote a closed symplectic manifold of dimension four. A closed hypersurface $M \subset W$ is of contact type if it is transverse to a Liouville vector field, ie a smooth vector field $Y$ defined near $M$ such that $L_{Y} \omega=\omega$. Then $\iota_{Y} \omega$ is a contact form on $M$, and we will denote the resulting contact structure by $\xi=\operatorname{ker} \iota_{Y} \omega$; it is independent of $Y$ up to isotopy. If $M$ separates $W$ into two components, then it is said to form a convex boundary on the component where $Y$ points outward, and a concave boundary on the other component. By constructions due to Etnyre-Honda [8] and Eliashberg [5], every contact 3-manifold can occur as the concave boundary of some compact symplectic manifold. This is not true for convex boundaries: for instance, Gromov [15] and Eliashberg [4] showed that overtwisted contact manifolds can never occur as convex boundaries, and a finer obstruction comes from Giroux torsion; see Gay [10].

In this paper, we address the question of whether a given contact 3-manifold $(M, \xi)$ can occur as a nonseparating contact hypersurface in any closed symplectic manifold, and similarly, whether a given symplectic 4 -manifold $(W, \omega)$ admits nonseparating 
contact hypersurfaces. Observe that separating contact hypersurfaces always exist in abundance, eg the boundaries of balls in Darboux neighborhoods. We will see in Example 1.3 that nonseparating contact hypersurfaces sometimes exist, but there are restrictions, as the following theorem shows.

Theorem 1.1 Suppose $(M, \xi)$ is a closed contact 3-manifold which has any one of the following properties:

(1) $(M, \xi)$ has Giroux torsion.

(2) $(M, \xi)$ is planar or partially planar (see Definition 1.9 below).

(3) $(M, \xi)$ admits a symplectic cap containing a symplectically embedded sphere of nonnegative self-intersection number.

Then every contact type embedding of $(M, \xi)$ into any closed symplectic 4-manifold is separating.

Remark 1.2 Theorem 1.1 admits an easy generalization as follows. We will say that $(M, \xi)$ has any given property after contact surgery if the property holds for some contact manifold $\left(M^{\prime}, \xi^{\prime}\right)$ obtained from $(M, \xi)$ by a (possibly trivial) sequence of contact connected sum operations and contact $(-1)$-surgeries. The significance of these operations (see eg Geiges [13]) is that they imply the existence of a symplectic cobordism from $(M, \xi)$ to $\left(M^{\prime}, \xi^{\prime}\right)$ : recall that a symplectic cobordism from $\left(M_{-}, \xi_{-}\right)$to $\left(M_{+}, \xi_{+}\right)$is in general a compact symplectic manifold $(W, \omega)$ with $\partial W=\left(-M_{-}\right) \sqcup M_{+}$, such that there is a Liouville vector field near $\partial W$ defining $\left(M_{-}, \xi_{-}\right)$and $\left(M_{+}, \xi_{+}\right)$as concave and convex boundary components respectively. The special case where $M_{-}=\varnothing$ is a convex filling of $\left(M_{+}, \xi_{+}\right)$. If $M_{+}=\varnothing$ we instead get a concave filling of $\left(M_{-}, \xi_{-}\right)$, also known as a symplectic cap.

It will follow from the more general Theorem 2.7 below that Theorem 1.1 also holds whenever properties (1) or (2) hold after contact surgery. (For property (3) this statement is trivial.)

The following example shows that nonseparating contact type hypersurfaces do exist in general.

Example 1.3 (Etnyre) Suppose $\left(W_{0}, \omega_{0}\right)$ is a compact symplectic manifold with a convex boundary that has two connected components. In this case we say that $\left(W_{0}, \omega_{0}\right)$ is a convex semifilling of each of its boundary components; the existence of such objects was first established by McDuff [27]. Produce a new symplectic manifold $\left(W_{1}, \omega_{1}\right)$ with convex boundary by attaching a symplectic 1-handle along a pair of 3-balls in 
different components of $\partial W_{0}$. Now cap $W_{1}$ with a concave filling of $\partial W_{1}$ as provided by Etnyre and Honda [8]: this produces a closed symplectic manifold $(W, \omega)$, which contains both of the components of $\partial W_{0}$ as nonseparating contact hypersurfaces (see Figure 1).
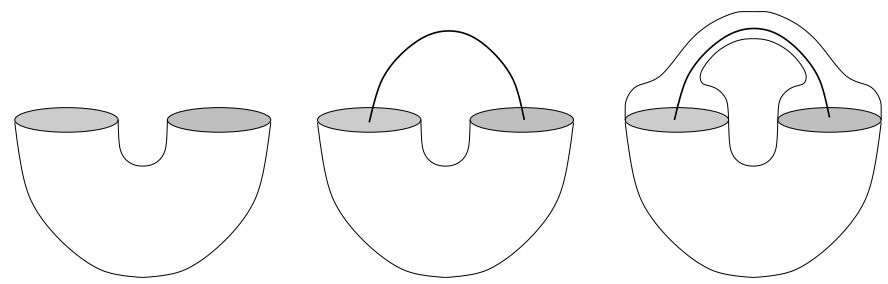

Figure 1: The construction from Example 1.3 of a symplectic manifold with nonseparating contact hypersurfaces

The example demonstrates that $(M, \xi)$ can occur as a nonseparating hypersurface in some closed symplectic manifold whenever it arises from a convex filling with disconnected boundary. There are, however, contact manifolds that never arise in this way: McDuff [27] showed that this is the case for the tight 3-sphere, and the result was generalized by Etnyre [6] to all planar contact manifolds, ie those which are supported by planar open books. The latter suggests that planar open books may provide an obstruction to nonseparating contact embeddings, and this is indeed true due to Theorem 1.1. As we'll see shortly, there are also nonplanar contact manifolds (eg the standard contact 3-torus) which satisfy the assumptions of Theorem 1.1, and thus also the following corollary:

Corollary 1.4 Given the assumptions of Theorem 1.1 (see also Remark 1.2), every convex semifilling of $(M, \xi)$ has connected boundary.

Actually one can use the same methods to give a slightly simpler proof of Corollary 1.4 which is independent of the theorem; we'll do this in Section 5.

In the case of Giroux torsion, a result of Gay [10] shows that $(M, \xi)$ does not admit any convex fillings, ${ }^{1}$ thus Theorem 1.1 has the following stronger consequence:

Corollary 1.5 If $(M, \xi)$ has Giroux torsion (possibly after contact surgery), then it does not admit a contact embedding into any closed symplectic 4-manifold.

\footnotetext{
${ }^{1}$ An alternative proof closely related to the arguments in this paper appears in Wendl [40].
} 
Theorem 1.1 will follow from some more technical results stated in Section 2.2, which also includes a more general statement involving contact hypersurfaces in a symplectic manifold with convex boundary. The unifying idea can be summarized as follows. Whenever a nonseparating hypersurface $M \subset W$ exists, one can use it to construct a special noncompact symplectic manifold $(\mathcal{V}, \omega)$ with convex boundary $M$. We do this by first cutting $W$ open along $M$ to produce a symplectic cobordism $\left(V_{1}, \omega\right)$ from a concave copy of $M$ to a convex copy of $M$, and then removing the concave boundary by attaching an infinite chain of copies of $\left(V_{1}, \omega\right)$ along matching concave and convex boundaries; a picture of this construction appears as Figure 4 in Section 5, where it is explained in detail. Now our assumptions on $(W, \omega)$ or $(M, \xi)$ guarantee the existence of an embedded holomorphic curve in $(\mathcal{V}, \omega)$ with certain properties: in particular, we'll show in Section 4 that this curve belongs to a smooth and compact 2 -dimensional moduli space of curves that foliate $(\mathcal{V}, \omega)$. But this would imply that $(\mathcal{V}, \omega)$ is compact, and thus yields a contradiction.

Remark 1.6 A contact manifold $(M, \xi)$ is said to be weakly fillable if it occurs as the boundary of a compact symplectic manifold $(W, \omega)$ such that $\left.\omega\right|_{\xi}>0$ on $\partial W$. A fundamental result of Eliashberg [4] and Gromov [15] shows that overtwisted contact manifolds are never weakly fillable: the original proof is based on the existence of a socalled Bishop family of pseudoholomorphic disks with boundary on an overtwisted disk in $\partial W$, and derives a contradiction using Gromov compactness (a complete exposition may be found in Zehmisch [42]). In the setting described above, one can adapt the Eliashberg-Gromov argument to show that overtwisted contact manifolds do not occur as hypersurfaces of weak contact type in any closed symplectic manifold. If we remove the word "weak", then this is also implied by Corollary 1.5 since overtwisted contact manifolds have infinite Giroux torsion.

The third condition in Theorem 1.1 is satisfied by any contact 3-manifold that has a contact embedding into the standard symplectic $\mathbb{R}^{4}$ : indeed, the latter can be identified with $\mathbb{C} P^{2} \backslash \mathbb{C} P^{1}$, and $\mathbb{C} P^{1}$ is a symplectically embedded sphere with self-intersection 1 . As Yasha Eliashberg has pointed out to us, Theorem 1.1 in this case also morally follows, via the infinite chain construction sketched above, from Gromov's classification [15] of symplectic manifolds that are Euclidean at infinity-one just has to be a little more careful in the noncompact setting (cf Proposition 5.3). Natural examples are the unit cotangent bundles of all closed surfaces that admit Lagrangian embeddings into $\mathbb{R}^{4}$, ie the torus, and the connected sums of the Klein bottle with a positive number of oriented surfaces of positive, even genus. Further examples of symplectic caps containing nonnegative symplectic spheres have appeared in the work of Ohta and Ono [30] and Bhupal and Ono [2] on contact manifolds obtained from algebraic surface singularities. 
We now explain the notion of a partially planar contact manifold, which is due to the third author (cf [37; 36]). Recall that an open book decomposition for $M$ consists of the data $(B, \pi)$ where $B \subset M$ is an oriented link, and $\pi: M \backslash B \rightarrow S^{1}$ is a fibration for which each fiber $\pi^{-1}$ (point) is an embedded surface whose closure in $M$ has oriented boundary $B$. These fibers are called the pages of the open book $(B, \pi)$, and $B$ is called the binding. We recall the following important concept introduced by Giroux [14].

Definition 1.7 A contact structure $\xi$ on $M$ is said to be supported by an open book decomposition $(B, \pi)$ if it admits a contact form $\lambda$ such that the associated Reeb vector field is positively transverse to the pages and is positively tangent to the link $B$.

In particular, the component circles of $B$ are closed Reeb orbits for such a contact form $\lambda$. These are referred to as the binding orbits.

Definition 1.8 A contact manifold $(M, \xi)$ is said to be planar if it admits a supporting open book decomposition for which each page has genus zero.

Giroux established that every contact structure on a closed 3-manifold is supported by some open book decomposition. Etnyre showed in [6] that all overtwisted contact structures are planar, though not all contact structures are.

The notion of a planar contact manifold can be generalized using the contact fiber sum; the following is a special case of a construction originally due to Gromov [16] and Geiges [12] (see also Geiges' book [13]). For $i=1,2$, suppose $\left(M_{i}, \xi_{i}\right)$ are contact manifolds with supporting open book decompositions $\pi_{i}: M_{i} \backslash B_{i} \rightarrow S^{1}$, and $\gamma_{i} \subset B_{i}$ are connected components of the bindings. Each $\gamma_{i}$ is a transverse knot, thus one can identify neighborhoods $\mathcal{N}\left(\gamma_{i}\right)$ with solid tori via an orientation preserving map

$$
\Phi: \mathcal{N}\left(\gamma_{1}\right) \cup \mathcal{N}\left(\gamma_{2}\right) \rightarrow S^{1} \times \mathbb{D},
$$

thus defining coordinates $(\theta, \rho, \phi)$, where $\theta \in S^{1}$ and $(\rho, \phi)$ are polar coordinates on $\mathbb{D}$ (for simplicity we shall take $\phi \in S^{1}=\mathbb{R} / \mathbb{Z}$, thus the actual angle is this times $2 \pi$ ). We will assume without loss of generality (and perhaps after a small isotopy of the open books) that these coordinates have the following properties:

(1) The contact structure $\xi_{i}$ is the kernel of $\lambda_{i}=f(\rho) d \theta+g(\rho) d \phi$ for some pair of functions $f$ and $g$ with $f(0)>0$ and $g(0)=0$.

(2) The pages of $\pi_{i}$ have the form $\{\phi=$ const $\}$ near $\gamma_{i}$. 
Note that the contact condition requires $f(\rho) g^{\prime}(\rho)-f^{\prime}(\rho) g(\rho)>0$ for $\rho>0$ and $g^{\prime \prime}(0)>0$. Using these choices, a new contact manifold

$$
\left(M_{1}, \xi_{1}\right) \#_{\Phi}\left(M_{2}, \xi_{2}\right)
$$

can be defined in two steps:

(i) Modify $\left(M_{i}, \xi_{i}\right)$ by "blowing up" $\gamma_{i}$ to produce a contact manifold $\left(\widehat{M}_{i}, \hat{\xi}_{i}\right)$ with pre-Lagrangian torus boundary: we do this by removing a solid torus neighborhood $\{\rho \leq \epsilon\}$ and replacing it with $S^{1} \times[0, \epsilon] \times S^{1}$ by the natural identification of the coordinates $(\theta, \rho, \phi) \in S^{1} \times[0, \epsilon] \times S^{1}$. We also modify $\lambda_{i}$ for $\rho \in[0, \epsilon)$ to define a smooth contact form near $\partial \widehat{M}_{i}$ by making $C^{0}$-small changes to $f$ and $g$ so that they become restrictions of even and odd functions respectively, with $g^{\prime}(0)>0$. In terms of the Reeb vector field defined by $\lambda_{i}$, the result of this change is to replace the single Reeb orbit originally at $\{\rho=0\}$ by a torus $S^{1} \times S^{1}$ foliated by Reeb orbits of the form $S^{1} \times\{\mathrm{pt}\}$.

(ii) Attach $\left(\widehat{M}_{1}, \hat{\xi}_{1}\right)$ to $\left(\widehat{M}_{2}, \widehat{\xi}_{2}\right)$ along their boundaries as follows: first, define new coordinates $(\hat{\theta}, \hat{\rho}, \widehat{\phi}) \in S^{1} \times \mathbb{R} \times S^{1}$ near $\partial \widehat{M}_{i}$ so that they are the same as the old coordinates on $\widehat{M}_{1}$, but on $\widehat{M}_{2}$ we set

$$
(\hat{\theta}, \hat{\rho}, \widehat{\phi}):=(\theta,-\rho,-\phi),
$$

so $\hat{\rho} \leq 0$ near $\partial \widehat{M}_{2}$. We now attach $\widehat{M}_{1}$ to $\widehat{M}_{2}$ via a diffeomorphism such that $(\hat{\theta}, \hat{\rho}, \widehat{\phi}) \in S^{1} \times[-\epsilon, \epsilon] \times S^{1}$ become well defined coordinates after attaching. Our assumptions on the modified functions $f$ and $g$ imply also that $f(\hat{\rho}) d \hat{\theta}+$ $g(\hat{\rho}) d \widehat{\phi}$ gives a smooth contact form on $M_{1} \#_{\Phi} M_{2}$ which matches the original outside the region $\{\hat{\rho} \in(-\epsilon, \epsilon)\}$.

In a straightforward way, one can generalize this definition to a sum of two or more open books on contact manifolds $\left(M_{1}, \xi_{1}\right), \ldots,\left(M_{N}, \xi_{N}\right)$ along multiple binding components: then each of these components becomes a boundary component in its respective "blown up" manifold $\widehat{M}_{i}$, and it becomes a special pre-Lagrangian torus in the sum

$$
\#_{\Phi}\left(M_{i}, \xi_{i}\right)
$$

Definition 1.9 We say that $(M, \xi)$ is partially planar if it can be constructed in the above manner as a contact fiber sum along binding orbits of open book decompositions, at least one of which is planar.

Obviously, every planar contact manifold is also partially planar. Since there exist contact 3-manifolds that admit semifillings with disconnected boundary, a consequence of Corollary 1.4 is now the following: 


\section{Corollary 1.10 Not every contact manifold is partially planar.}

Example 1.11 McDuff showed in [27] that for any closed oriented surface $\Sigma$ of genus at least two, if $S T^{*} \Sigma$ denotes the unit cotangent bundle, then there is a symplectic structure on $[0,1] \times S T^{*} \Sigma$ which is convex on the boundary and induces the canonical contact structure at $\{1\} \times S T^{*} \Sigma$. More generally, Geiges [11] constructed a class of closed 3-manifolds $M$ which admit pairs of contact forms $\lambda_{ \pm}$such that

$$
\lambda_{+} \wedge d \lambda_{+}=-\lambda_{-} \wedge d \lambda_{-}>0 \quad \text { and } \quad \lambda_{+} \wedge d \lambda_{-}=\lambda_{-} \wedge d \lambda_{+}=0 .
$$

In this situation, $[0,1] \times M$ admits a symplectic structure such that both boundary components are convex, giving a convex filling of $\left(M, \operatorname{ker} \lambda_{+}\right) \sqcup\left(-M, \operatorname{ker} \lambda_{-}\right)$. It follows from Corollary 1.4 that none of these contact manifolds are partially planar. Moreover by Example 1.3, each of them admits a nonseparating contact type embedding into some closed symplectic manifold.

The next example shows that there are also partially planar contact manifolds that are not planar.

Example 1.12 The standard contact $S^{1} \times S^{2}$ is planar: it admits a supporting open book decomposition with two binding orbits connected by cylindrical pages. If we take two copies of this, pair up both of their respective binding components and construct the fiber sum, we obtain the standard contact $T^{3}$, which is not planar due to a result of Etnyre [6]. In fact, each of the tight contact tori $\left(T^{3}, \xi_{n}\right)$, where $\xi_{n}=$ $\operatorname{ker}(\cos (2 \pi n \theta) d x+\sin (2 \pi n \theta) d y)$ in coordinates $(x, y, \theta) \in S^{1} \times S^{1} \times S^{1}$, can be obtained as a fiber sum of $2 n$ copies of the standard $S^{1} \times S^{2}$; see Figure 2. By a result of Kanda [24], this includes every tight contact structure on $T^{3}$.

Remark 1.13 As pointed out to us by Etnyre, one can often interpret the summing procedure described above in terms of open books with quasi-compatible contact structures, a notion defined by Etnyre and Van Horn-Morris [9]. This is true for instance with $T^{3}$ as in Example 1.12, whose tight contact structures are all quasicompatible with the natural fibration $T^{3} \rightarrow S^{1}$ (viewed as an open book with empty binding) obtained by piecing together its constituent planar open books. This only works however if a certain orientation condition is satisfied, eg one obtains no such fibration after doing the trick in Example 1.12 with an odd number of copies of $S^{1} \times S^{2}$.

By Example 1.12, every contact structure on $T^{3}$ is partially planar. In fact, other than the standard torus $\left(T^{3}, \xi_{1}\right)$, all contact 3 -tori also have Giroux torsion, thus $\xi_{1}$ is the only convex fillable contact structure on $T^{3}$. Theorem 1.1 therefore implies that every 

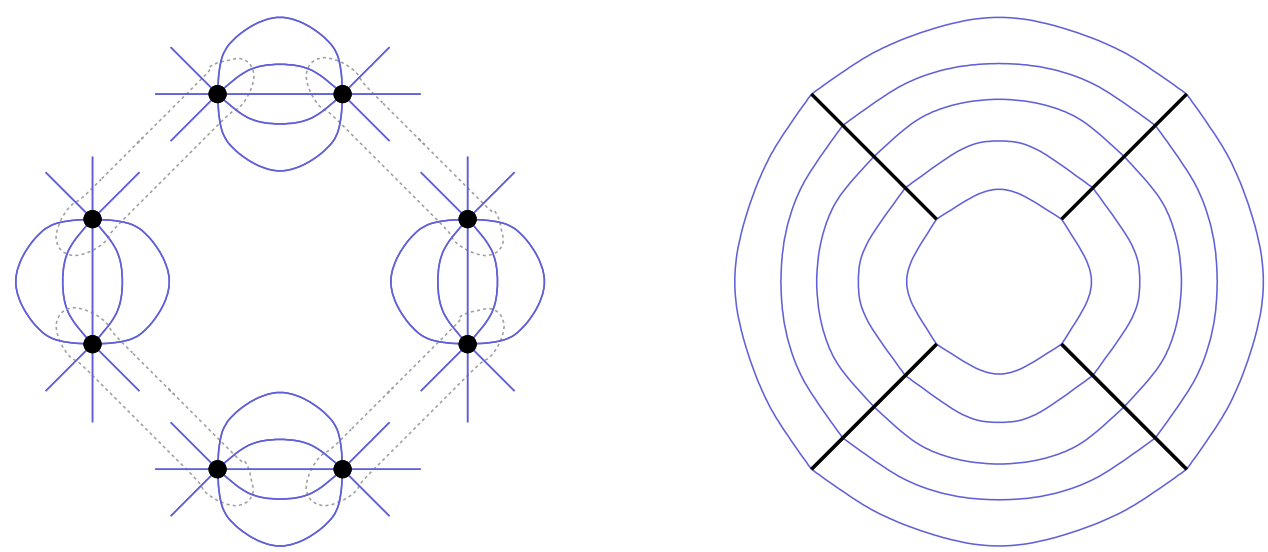

Figure 2: At left, we see four copies of the tight $S^{1} \times S^{2}$, represented by open books with two binding components and cylindrical pages. For each dotted oval surrounding two binding components, we construct the contact fiber sum to produce the manifold at right, containing four special pre-Lagrangian tori (the black line segments) that separate regions foliated by cylinders. The result is the tight 3 -torus $\left(T^{3}, \xi_{2}\right)$. In general, one can construct $\left(T^{3}, \xi_{n}\right)$ from $2 n$ copies of the tight $S^{1} \times S^{2}$.

contact type embedding of $T^{3}$ into a closed symplectic 4-manifold separates (and the induced contact structure must be $\xi_{1}$ ). This result is not true for embeddings of weak contact type: in fact all of the tight tori $\left(T^{3}, \xi_{n}\right)$ admit weak symplectic semifillings with disconnected boundary [7], and thus by the construction in Example 1.3, they also admit nonseparating weakly contact type embeddings.

Recall however that if $(W, \omega)$ is a weak filling of $(M, \xi)$ and $M$ is a homology 3sphere, then $\omega$ can always be deformed in a collar neighborhood of $\partial W$ to produce a convex filling of $(M, \xi)$; see for instance Geiges [13, Lemma 6.5.5]. Thus our results have corresponding versions for weakly contact hypersurfaces that are homology 3spheres. For example, since the only tight contact structure on $S^{3}$ is planar, every weakly contact type embedding of $S^{3}$ into a closed symplectic 4-manifold must separate.

Here is a more general example that also implies the observation made above about the 3-torus. Let

$$
\Sigma=\Sigma_{+} \cup_{\Gamma} \Sigma_{-}
$$

denote any closed oriented surface obtained as the union of two nonempty surfaces with boundary $\Sigma_{ \pm}$along a multicurve $\Gamma \subset \Sigma$. By a result of Lutz [25], the manifold $M_{\Gamma}:=S^{1} \times \Sigma$ admits a unique (up to isotopy) $S^{1}$-invariant contact structure $\xi_{\Gamma}$ which 
makes $\Gamma$ the dividing set on $\{$ const $\} \times \Sigma$. We claim that $\left(M_{\Gamma}, \xi_{\Gamma}\right)$ is partially planar whenever there exists a connected component of $\Sigma \backslash \Gamma$ having genus zero. Indeed, for any connected component $\Sigma_{0} \subset \Sigma \backslash \Gamma$, the closure of $S^{1} \times \Sigma_{0}$ may be viewed as an open book with page $\Sigma_{0}$ and trivial monodromy, blown up at all its binding circles; the entirety of $\left(M_{\Gamma}, \xi_{\Gamma}\right)$ can thus be obtained by attaching these blown up open books. (The tight 3-tori arise from the case where $\Sigma \cong T^{2}$ and $\Gamma$ is a union of parallel curves that are primitive in $H_{1}\left(T^{2}\right)$.) Moreover, using Etnyre's obstruction [6] it is easy to construct many examples $\left(M_{\Gamma}, \xi_{\Gamma}\right)$ which are partially planar (as just explained) but not planar. Theorem 1.1 now implies:

Corollary 1.14 If $\Sigma \backslash \Gamma$ has a connected component of genus zero, then the $S^{1}-$ invariant contact manifold $\left(S^{1} \times \Sigma, \xi_{\Gamma}\right)$ does not admit any nonseparating contact type embeddings into closed symplectic 4 -manifolds.

Finally, the following demonstrates that in some settings where nonseparating hypersurfaces can be embedded smoothly, they can never be contact type. In contrast to Theorem 1.1, here the assumptions are on the ambient symplectic 4-manifold and not the contact manifold.

Theorem 1.15 If the closed and connected symplectic 4-manifold $(W, \omega)$ contains a symplectically embedded sphere $S \subset W$ with self-intersection number $S \bullet S \geq 0$, then every closed contact type hypersurface in $W$ is separating.

The reason for this is closely related to McDuff's results [26], which imply that $(W, \omega)$ in this situation is always rational or ruled (up to symplectic blowup). In fact, the case where $S \bullet S>0$ follows immediately from [26], which shows that $W$ is then a blowup of either $S^{2} \times S^{2}$ or $\mathbb{C} P^{2}$ and thus simply connected, so it does not admit nonseparating hypersurfaces at all (contact or otherwise). The case $S \bullet S=0$ is more interesting: the key fact here is that one can choose a compatible almost complex structure $J$ for which any given contact hypersurface $M \subset W$ is $J$-convex, and $W$ is foliated by a family of embedded $J$-holomorphic spheres (possibly including some isolated nodal spheres unless $(W, \omega)$ is minimal). If $M$ does not separate, then there exists a connected infinite cover $(\widetilde{W}, \widetilde{J})$ of $(W, J)$, constructed by gluing together infinitely many copies of $W \backslash M$ in a sequence. Now the $J$-holomorphic spheres in $W$ lift to $\widetilde{W}$ and form a foliation, which must include a $J$-holomorphic sphere that touches a lift of $M$ tangentially from below, violating $J$-convexity. That's a quick sketch of the proof-we'll give an alternative proof in Section 5 that fits into a usefully generalized context and doesn't assume the results of [26]. There are obvious examples of smoothly embedded nonseparating hypersurfaces in ruled surfaces, eg $\ell \times S^{2} \subset \Sigma \times S^{2}$, where $\Sigma$ is any closed oriented surface of positive genus and $\ell \subset \Sigma$ is a nonseparating closed curve. It follows that a hypersurface isotopic to this one is never contact type. 


\subsection{Open questions}

Let $\Xi$ (3) denote the collection of closed 3-manifolds with positive, cooriented contact structures, and consider the inclusions

$$
\Xi_{\text {nonsep }}(3) \subsetneq \Xi_{\text {embed }}(3) \subsetneq \Xi(3),
$$

where $\Xi_{\text {embed }}(3)$ denotes all $(M, \xi) \in \Xi(3)$ that admit a contact type embedding into some closed symplectic manifold, and $\Xi_{\text {nonsep }}(3)$ denotes those that admit a nonseparating embedding. The results stated in Section 1.1 imply that both inclusions are proper.

Observe that if $(M, \xi)$ is convex fillable then it is also in $\Xi_{\text {embed }}(3)$, since a filling can always be capped to produce a closed symplectic manifold. Conversely, if $(M, \xi)$ admits a separating contact type embedding, then it is fillable. While the same is not strictly true for a nonseparating embedding, the construction depicted in Figure 4 of Section 5 can be viewed as a filling that is noncompact but geometrically bounded, which makes it a good setting for $J$-holomorphic curves. In this context, any filling obstruction that involves $J$-holomorphic curves can also serve as an obstruction to nonseparating contact embeddings (cf Corollary 1.5), thus implying that $(M, \xi) \notin \Xi_{\text {embed }}(3)$. This motivates the conjecture that, in fact, $\Xi_{\text {embed }}(3)$ is the same as the set of convex fillable contact 3-manifolds.

Conjecture 1 If $(M, \xi)$ is not convex fillable, then it admits no contact type embeddings into any closed symplectic manifold.

Equivalently, this would mean there is no contact 3-manifold that admits only nonseparating contact type embeddings.

A more ambitious conjecture would arise from Example 1.3, which is the only method we are yet aware of for constructing nonseparating contact embeddings: $(M, \xi) \in$ $\Xi_{\text {nonsep }}(3)$ whenever it admits a convex semifilling with disconnected boundary. The latter class of contact manifolds is evidently somewhat special, and one wonders whether it might be equal to $\Xi_{\text {nonsep }}(3)$.

Question 1 Is there a contact 3-manifold that admits a nonseparating contact type embedding but not a convex semifilling with disconnected boundary?

Finally, observe that while Theorem 1.15 rules out the existence of a nonseparating contact hypersurface $(M, \xi) \subset(W, \omega)$ if $(W, \omega)$ is rational or ruled, it still allows the possibility that $(M, \xi) \in \Xi_{\text {nonsep }}(3)$ but admits a separating embedding into $(W, \omega)$. 
There is some reason to suspect that this could still never happen. There are indeed cases where the existence of a contact embedding of $(M, \xi)$ into some particular symplectic manifold implies $(M, \xi) \notin \Xi_{\text {nonsep }}(3)$, eg this is true if $(M, \xi) \hookrightarrow\left(\mathbb{R}^{4}, \omega_{0}\right)$. Moreover, the simplest known example of a manifold in $\Xi_{\text {nonsep }}(3)$, the unit cotangent bundle of a higher genus surface, has been shown by Welschinger [33] to admit no contact type embeddings into rational or ruled symplectic 4-manifolds.

Question 2 Is there a contact 3-manifold that admits a contact type embedding into some rational/ruled symplectic 4-manifold and also admits a nonseparating contact type embedding into some other closed symplectic manifold?

\section{Pseudoholomorphic curves in symplectizations}

\subsection{Technical background}

In this section we collect a number of important technical definitions. A positive contact form on a 3 -manifold $M$ is a 1 -form $\lambda$ for which $\lambda \wedge d \lambda>0$. The 2plane distribution $\xi:=\operatorname{ker} \lambda$ is then a contact structure. The equations $\iota_{X_{\lambda}} d \lambda=0$ and $\lambda\left(X_{\lambda}\right)=1$ uniquely determine a vector field $X_{\lambda}$, called the Reeb vector field associated to $\lambda$. Since $X_{\lambda}$ is everywhere transverse to $\xi$, one obtains a splitting $T M=\mathbb{R} X_{\lambda} \oplus \xi$. Moreover, $\left(\xi,\left.d \lambda\right|_{\xi}\right)$ is a symplectic vector bundle, and the flow of $X_{\lambda}$ preserves $\lambda$, hence also $\left(\xi,\left.d \lambda\right|_{\xi}\right)$.

A periodic Reeb orbit of period $T>0$ for a contact form $\lambda$ is a smooth map $\gamma: \mathbb{R} / T \mathbb{Z} \rightarrow M$ satisfying $\dot{\gamma}(t)=X_{\lambda}(\gamma(t))$. We identify all possible reparametrizations $t \mapsto \gamma(t+$ const $)$. A Reeb orbit is called simply covered if it has degree 1 onto its image, ie it is an embedding. If $\gamma$ covers a simply covered orbit with period $\tau>0$, we call $\tau$ the minimal period of $\gamma$.

Since the Reeb flow preserves the symplectic vector bundle $\left(\xi,\left.d \lambda\right|_{\xi}\right)$, linearizing about a periodic orbit $\gamma$ determines a symplectic linear map $d \phi_{T}(p): \xi_{p} \rightarrow \xi_{p}$ for each $p$ in the image of $\gamma$. Then $\gamma$ is said to be nondegenerate if 1 is not an eigenvalue of this map; this condition is independent of the point $p$. More generally, an orbit $\gamma$ of period $T$ is Morse-Bott if it lies in a submanifold $N \subset M$ foliated by $T$-periodic orbits, such that the 1-eigenspace of $d \phi_{T}(p)$ is precisely $T_{p} N$. We then call $N$ a Morse-Bott submanifold. A contact form $\lambda$ is said to be nondegenerate if all of its periodic Reeb orbits are nondegenerate, and Morse-Bott if every periodic orbit belongs to a Morse-Bott submanifold.

Given a symplectic trivialization $\Phi$ of $(\xi, d \lambda)$ along a $T$-periodic orbit $\gamma$, the linearized flow $d \phi_{t}(p)$ for $t \in[0, T]$ defines a continuous family of symplectic matrices, 
which has a well defined Conley-Zehnder index if $\gamma$ is nondegenerate: we denote this index by $\mu_{\mathrm{CZ}}^{\Phi}(\gamma) \in \mathbb{Z}$.

It is convenient also to express this in terms of asymptotic operators: associated to any $T$-periodic Reeb orbit $\gamma$ is a linear operator $\mathbf{A}_{\gamma}: \Gamma\left(\mathbf{x}^{*} \xi\right) \rightarrow \Gamma\left(\mathbf{x}^{*} \xi\right)$, where $\mathbf{x}: \mathbb{R} / \mathbb{Z} \rightarrow M$ is the reparametrization $\mathbf{x}(t):=\gamma(T t)$. If $\nabla$ is a symmetric connection on $T M$ and $J$ is a complex structure on $\xi \rightarrow M$ compatible with the symplectic structure $\left.d \lambda\right|_{\xi}$, then $\mathbf{A}_{\gamma}$ can be defined on smooth sections by

$$
\mathbf{A}_{\gamma} \eta=-J\left(\nabla_{t} \eta-T \nabla_{\eta} X_{\lambda}\right)
$$

This expression is independent of the choice of connection. Choosing a unitary trivialization $\Phi$ of $\mathbf{x}^{*} \xi, \mathbf{A}_{\gamma}$ is identified with the operator

$$
C^{\infty}\left(S^{1}, \mathbb{R}^{2}\right) \rightarrow C^{\infty}\left(S^{1}, \mathbb{R}^{2}\right): \eta \mapsto-J_{0} \frac{d}{d t} \eta-S \cdot \eta,
$$

where $S(t)$ is some smooth loop of symmetric 2-by-2 matrices. Thus the equation $\mathbf{A}_{\gamma} \eta=0$ defines a linear Hamiltonian flow, and one can show that the resulting family of symplectic matrices matches the family obtained from $d \phi_{t}(p)$. It follows that $\mathbf{A}_{\gamma}$ has trivial kernel if and only if $\gamma$ is nondegenerate, and we can use the linear Hamiltonian flow determined by (2-1) to define an integer $\mu_{\mathrm{CZ}}^{\Phi}\left(\mathbf{A}_{\gamma}\right)$, which matches $\mu_{\mathrm{CZ}}^{\Phi}(\gamma)$. The advantage of this definition is that it does not reference the orbit directly, but makes sense for any operator that takes the form of (2-1) in the trivialization: in particular we can define $\mu_{\mathrm{CZ}}^{\Phi}\left(\mathbf{A}_{\gamma}-c\right) \in \mathbb{Z}$ whenever $c \in \mathbb{R}$ is not an eigenvalue of $\mathbf{A}_{\gamma}$, even if $\gamma$ is degenerate. For this we will use the shorthand notation

$$
\mu_{\mathrm{CZ}}^{\Phi}(\gamma-c):=\mu_{\mathrm{CZ}}^{\Phi}\left(\mathbf{A}_{\gamma}-c\right) .
$$

We now recall some of the important spectral properties of asymptotic operators. For more details and proofs we refer to Hofer, Wysocki and Zehnder [19].

$\mathbf{A}_{\gamma}$ extends to an unbounded self-adjoint operator on the complexified Hilbert space $L^{2}\left(\mathbf{x}^{*} \xi\right)$; its spectrum $\sigma\left(\mathbf{A}_{\gamma}\right)$ consists of real eigenvalues of multiplicity at most 2 that accumulate only at infinity. Generalizing the statement above about nondegeneracy, if $\gamma$ belongs to a Morse-Bott submanifold of dimension $n \in\{1,2,3\}$, then the 0 -eigenspace of $\mathbf{A}_{\gamma}$ is $(n-1)$-dimensional.

Geometric properties of the eigenspaces are closely related to the Conley-Zehnder index. Indeed, any eigenfunction $\eta$ of $\mathbf{A}_{\gamma}$ has a well defined winding number wind ${ }^{\Phi}(\eta) \in \mathbb{Z}$ relative to the trivialization, which is independent of the choice of $\eta$ in its eigenspace. Thus we may speak of the winding number wind ${ }^{\Phi}(\mu) \in \mathbb{Z}$ for each eigenvalue $\mu \in$ $\sigma\left(\mathbf{A}_{\gamma}\right)$, and it turns out that the map $\sigma\left(\mathbf{A}_{\gamma}\right) \rightarrow \mathbb{Z}: \mu \mapsto$ wind $^{\Phi}(\mu)$ is nondecreasing 
and attains every value exactly twice (counting multiplicity). The following integers

$$
\begin{aligned}
& \alpha_{-}^{\Phi}(\gamma):=\max \left\{\operatorname{wind}^{\Phi}(\mu) \mid \mu<0 \text { is an eigenvalue of } \mathbf{A}_{\gamma}\right\} \\
& \alpha_{+}^{\Phi}(\gamma):=\min \left\{\text { wind }^{\Phi}(\mu) \mid \mu>0 \text { is an eigenvalue of } \mathbf{A}_{\gamma}\right\}
\end{aligned}
$$

are therefore determined by the eigenfunctions with eigenvalues closest to 0 that are negative and positive respectively. The number $p(\gamma):=\alpha_{+}^{\Phi}(\gamma)-\alpha_{-}^{\Phi}(\gamma)$ is called the parity of $\gamma$; it is independent of $\Phi$ and necessarily equals 0 or 1 if $\gamma$ is nondegenerate. More generally, we can replace $\mathbf{A}_{\gamma}$ by $\mathbf{A}_{\gamma}-c$ for some $c \in \mathbb{R}$ and similarly define $\alpha_{ \pm}^{\Phi}(\gamma-c)$ and $p(\gamma-c)$; then if $c \notin \sigma\left(\mathbf{A}_{\gamma}\right)$, a result in [19] implies the relation

$$
\mu_{\mathrm{CZ}}^{\Phi}(\gamma-c)=2 \alpha_{-}^{\Phi}(\gamma-c)+p(\gamma-c)=2 \alpha_{+}^{\Phi}(\gamma-c)-p(\gamma-c) .
$$

Observe that every Morse-Bott submanifold of dimension 2 admits a nonzero vector field and is thus either a torus or a Klein bottle. The following characterization of Morse-Bott tori is a simple consequence of the spectral properties of $\mathbf{A}_{\gamma}$ (cf [34, Proposition 4.1]).

Proposition 2.1 Suppose $\gamma$ is a Morse-Bott periodic orbit of $X_{\lambda}$ belonging to a Morse-Bott submanifold $N \subset M$ diffeomorphic to $T^{2}$. Then the Morse-Bott property is satisfied for all covers of all orbits in $N$, and they all have the same minimal period.

We will also need a relative version of the standard genericity result for nondegenerate contact forms.

Lemma 2.2 Suppose $N \subset M$ is a union of 2-tori which are Morse-Bott submanifolds for some contact form $\lambda_{0}$. Then for any $T_{0}>0$, there exists an arbitrarily small perturbation $\lambda$ of $\lambda_{0}$ such that $\lambda=\lambda_{0}$ on a neighborhood of $N$ and every periodic orbit of $X_{\lambda}$ with period less than $T_{0}$ is Morse-Bott.

Proof Since all orbits in $N$ are Morse-Bott (including all multiple covers, due to Proposition 2.1), for any $T_{0}>0$ we can find an open neighborhood $\mathcal{U}$ of $N$ such that $\overline{\mathcal{U}} \backslash N$ contains no periodic orbits with period less than $T_{0}$. By Theorem A.1 in the Appendix, one can then find a generic small perturbation of $\lambda_{0}$ with support in $M \backslash \mathcal{U}$ so that all orbits passing through $M \backslash \overline{\mathcal{U}}$ are nondegenerate.

We now recall the basic notions of holomorphic curves in symplectizations and their asymptotic properties. The symplectization of a contact manifold $(M, \xi=\operatorname{ker} \lambda)$ is the product space $\mathbb{R} \times M$ equipped with the exact symplectic form $d\left(e^{a} \lambda\right)$, where $a: \mathbb{R} \times M \rightarrow \mathbb{R}$ refers to the $\mathbb{R}$ coordinate. An almost complex structure $J$ on the symplectization is said to be admissible if it is $\mathbb{R}$-invariant, restricts to the symplectic 
vector bundle $(\xi, d \lambda)$ as a compatible complex structure, and satisfies $J \partial_{a}=X_{\lambda}$. Any admissible $J$ tames the symplectic form $d\left(e^{a} \lambda\right)$, and more generally tames every symplectic form $d(\varphi \lambda)$ where $\varphi: \mathbb{R} \rightarrow(0, \infty)$ is smooth with $\varphi^{\prime}>0$.

A pseudoholomorphic (or $J$-holomorphic or simply holomorphic) curve from a punctured Riemann surface $(\dot{\Sigma}, j)$, into an almost complex manifold $(W, J)$ is a solution $u: \dot{\Sigma} \rightarrow W$ to the nonlinear Cauchy-Riemann equation $T u \circ j=J(u) \circ T u$. Here we take $\dot{\Sigma}:=\Sigma \backslash \Gamma$ for some finite set of points $\Gamma \subset \Sigma$, where $(\Sigma, j)$ is a closed connected Riemann surface.

For the rest of this section, let us consider only the case where the target is the symplectization of $(M, \lambda)$, and $J$ is an admissible almost complex structure on $\mathbb{R} \times M$. The simplest case of a punctured $J$-holomorphic curve in this setting is the so-called trivial cylinder

$$
u: S^{2} \backslash\{0, \infty\} \cong \mathbb{R} \times S^{1} \rightarrow \mathbb{R} \times M:(s, t) \mapsto(T s, \gamma(T t)),
$$

where $T>0$ and $\gamma$ is any $T$-periodic Reeb orbit. Following [17; 3], the energy of a $J$-holomorphic curve $u$ : $\dot{\Sigma} \rightarrow \mathbb{R} \times M$ can be defined as follows. Fix any constant $C>0$, and let

$$
E(u):=\sup _{\varphi \in \mathcal{T}} \int_{\dot{\Sigma}} u^{*} d(\varphi \lambda)
$$

where $\mathcal{T}$ is the set of smooth maps $\varphi: \mathbb{R} \rightarrow(0, C)$ with $\varphi^{\prime}>0$. Since $J$ is compatible with $d(\varphi \lambda)$ for all $\varphi \in \mathcal{T}$, the integrand in (2-3) is always nonnegative, thus $u$ is constant if and only if its energy vanishes. Observe that the integrand of $\int_{\dot{\Sigma}} u^{*} d \lambda$ is also nonnegative, and this integral is finite if $u$ has finite energy: it vanishes identically if and only if $u$ is a branched cover of a trivial cylinder.

Definition 2.3 We will say that $u$ : $\dot{\Sigma} \rightarrow \mathbb{R} \times M$ is a finite energy $J$-holomorphic curve if it is proper and $E(u)<\infty$.

Note that properness only fails when there exist punctures having neighborhoods which are mapped into a compact set, in which case these punctures can be removed by Gromov's removable singularity theorem. Since $d(\varphi \lambda)$ is exact, Stokes' theorem implies that not all punctures are removable unless $u$ is constant.

Let us recall now the behaviour of a finite energy $J$-holomorphic curve $u: \dot{\Sigma} \rightarrow \mathbb{R} \times M$ in the neighborhood of a puncture. Each puncture $z \in \Gamma$ has a neighborhood on which the $\mathbb{R}$-value of $u$ tends to $+\infty$ or $-\infty$, and we say that $z$ is a positive/negative puncture respectively. Denote the resulting partition into positive and negative punctures by $\Gamma=\Gamma^{+} \cup \Gamma^{-}$. Restricting to a neighborhood of a puncture, we obtain a curve whose 
domain is the punctured closed disc, which is biholomorphic to both $Z^{+}:=[0, \infty) \times S^{1}$ and $Z^{-}:=(-\infty, 0] \times S^{1}$ with the standard complex structure. It is convenient to choose the domain of the restricted curve to be $Z^{+}$or $Z^{-}$for $z \in \Gamma^{+}$or $z \in \Gamma^{-}$ respectively, and we will write $u: Z^{ \pm} \rightarrow \mathbb{R} \times M$. It was shown by Hofer in [17] that for any sequence $\left|s_{k}\right| \rightarrow \infty$, there exists a subsequence such that $u\left(s_{k}, \cdot\right)$ converges in $C^{\infty}\left(S^{1}, M\right)$ to $\gamma(T \cdot)$, where $\gamma$ is a $T$-periodic Reeb orbit for some $T>0$. We say in this case that $u$ is asymptotic to $\gamma$, and $\gamma$ is an asymptotic orbit of $u$.

In the following statement, we choose any $\mathbb{R}$-invariant connection on $\mathbb{R} \times M$ to define the exponential map, and use the term asymptotically trivial coordinates to refer to a diffeomorphism $(\sigma, \tau): Z^{ \pm} \rightarrow Z^{ \pm}$such that $\sigma(s, t)-s$ and $\tau(s, t)-t$ approach constants as $|s| \rightarrow \infty$ and their derivatives of all orders decay to zero.

Theorem $[21 ; 20 ; 29]$ Suppose $u: Z^{ \pm} \rightarrow \mathbb{R} \times M$ has finite energy and is asymptotic to a Morse-Bott Reeb orbit $\gamma$ of period $T>0$. Then there exist asymptotically trivial coordinates $(\sigma, \tau)$ such that for sufficiently large $|\sigma|$, either $u(\sigma, \tau)=(T \sigma, \gamma(T \tau))$ or

$$
u(\sigma, \tau)=\exp _{(T \sigma, \gamma(T \tau))}\left(e^{\mu \sigma}\left(e_{\mu}(\tau)+r(\sigma, \tau)\right)\right),
$$

where $e_{\mu}$ is an eigenfunction of $\mathbf{A}_{\gamma}$ with eigenvalue $\mu \in \sigma\left(\mathbf{A}_{\gamma}\right)$ such that $\pm \mu<0$, and the "remainder" term $r(\sigma, \tau) \in \xi_{\gamma(T \sigma)}$ decays to zero uniformly with all derivatives as $|\sigma| \rightarrow \infty$.

Definition 2.4 When (2-4) holds, we call $e_{\mu}$ the asymptotic eigenfunction of $u$ at the puncture, and say that $u$ has transversal convergence rate $|\mu|$. In the case where $u(\sigma, \tau)=(T \sigma, \gamma(T \tau))$, we define the asymptotic eigenfunction to be 0 and the transversal convergence rate to be $\infty$.

Observe that the asymptotic eigenfunction $e_{\mu}$ is determined uniquely once a parametrization of $\gamma$ is fixed. We know also from the monotonicity of winding numbers that wind $^{\Phi}\left(e_{\mu}\right) \leq \alpha_{-}^{\Phi}(\gamma)$ if the puncture is positive, and wind ${ }^{\Phi}\left(e_{\mu}\right) \geq \alpha_{+}^{\Phi}(\gamma)$ if it is negative.

Let $\pi_{\lambda}: T M \rightarrow \xi$ denote the natural projection with respect to the splitting $T M=$ $\mathbb{R} X_{\lambda} \oplus \xi$ and suppose $u=\left(u_{\mathbb{R}}, u_{M}\right): \dot{\Sigma} \rightarrow \mathbb{R} \times M$ is a finite energy $J$-holomorphic curve. Then the composition $\pi_{\lambda} \circ T u_{M}$ defines a section of the bundle of complex linear homomorphisms $(T \dot{\Sigma}, j) \rightarrow\left(u^{*} \xi, J\right)$. As shown in [19], this section satisfies a linear Cauchy-Riemann type equation, and thus is either trivial or has a discrete set of zeros, all of positive order. The former holds if and only if any asymptotic eigenfunction of $u$ vanishes, in which case they all do: then $\int_{\dot{\Sigma}} u^{*} d \lambda=0$ and $u$ is 
a branched cover of a trivial cylinder. Otherwise, (2-4) implies that $\pi_{\lambda} \circ T u_{M}$ has finitely many zeros, and we denote the algebraic count of these by

$$
\operatorname{wind}_{\pi}(u) \in \mathbb{Z} .
$$

Clearly $\operatorname{wind}_{\pi}(u) \geq 0$, with equality if and only if $u_{M}: \dot{\Sigma} \rightarrow M$ is an immersion.

\subsection{Property ( $\star$ ) and the main results}

We now use holomorphic curves to define two technical conditions on contact manifolds which imply the results stated in Section 1. Property ( $\star$ ) and its weak version, introduced below, will serve as obstructions to the existence of nonseparating contact embeddings. They are implied by each of the contact topological assumptions mentioned in Theorem 1.1, and in fact are more general (see also Wendl [37]).

Definition 2.5 A closed three-dimensional contact manifold $(M, \xi)$ satisfies Property $(\star)$ if there exists a contact form $\lambda$ with $\operatorname{ker} \lambda=\xi$ and an admissible $\mathbb{R}$-invariant almost complex structure $J$ on the symplectization $\mathbb{R} \times M$, which admits a finite energy $J$-holomorphic punctured sphere

$$
u=\left(u_{\mathbb{R}}, u_{M}\right): \dot{\Sigma}=S^{2} \backslash\left\{z_{1}, \ldots, z_{N}\right\} \rightarrow \mathbb{R} \times M
$$

with the following properties:

(1) $u_{M}$ is an embedding, and the closure of $u_{M}(\dot{\Sigma}) \subset M$ is an embedded surface whose oriented boundary is a union of Reeb orbits, called the "asymptotic orbits" of $u$.

(2) Each asymptotic orbit of $u$ is nondegenerate or Morse-Bott.

(3) If $T_{1}, \ldots, T_{N}$ are the periods of the asymptotic orbits of $u$, then every Reeb orbit not in the same Morse-Bott submanifold with one of these has period strictly greater than $T_{1}+\cdots+T_{N}$.

(4) $u$ has no asymptotic orbit that is nondegenerate with Conley-Zehnder index zero, relative to the natural trivialization determined by the image of $u_{M}$ near the puncture.

(5) If any asymptotic orbit of $u$ belongs to a 2-dimensional Morse-Bott manifold $N \subset M$ disjoint from $u_{M}(\dot{\Sigma})$, then $N$ is a torus and contains no other asymptotic orbits of $u$. 
Remarks - The fact that Reeb orbits comprise the oriented boundary of $\overline{u_{M}(\dot{\Sigma})}$ implies that every puncture of $u$ is positive. Moreover, each puncture is asymptotic to a distinct Reeb orbit, which is simply covered.

- The asymptotic formula (2-4) implies that on each cylindrical end of $\dot{\Sigma}, u_{M}$ does not intersect the corresponding asymptotic orbit, thus it defines a natural trivialization of $\xi$ along this orbit. One can then show (cf (2-2)) that relative to this trivialization, the orbit always has nonnegative Conley-Zehnder index if it is nondegenerate- thus our definition requires this index to be anything strictly larger than the minimum possible value.

Definition 2.6 We say that a closed three-dimensional contact manifold $(M, \xi)$ satisfies Weak Property $(\star)$ if there is a symplectic cobordism $(W, \omega)$ from $(M, \xi)$ to a contact manifold $\left(M^{\prime}, \xi^{\prime}\right)$, such that either $(W, \omega)$ contains a symplectically embedded sphere of nonnegative self-intersection number or $\left(M^{\prime}, \xi^{\prime}\right)$ satisfies Property $(\star)$.

For example, $(M, \xi)$ satisfies Weak Property $(\star)$ if it admits a symplectic cap containing a nonnegative symplectic sphere, or if it can be made to satisfy Property $(\star)$ after a sequence of contact $(-1)$-surgeries or connected sum operations. Obviously Property $(\star)$ implies Weak Property $(\star)$, and it's plausible that the converse may also be true, though this is presumably hard to prove.

We can now state some more technical results that imply Theorem 1.1. These will be proved in Section 5, using the machinery of Section 4.

Theorem 2.7 Let $(W, \omega)$ be a closed and connected symplectic 4-manifold which contains a closed contact type hypersurface $M \subset W$ satisfying Weak Property $(\star)$. Then $M$ separates $W$.

Theorem 2.8 Let $(W, \omega)$ be a compact and connected symplectic 4-manifold with convex boundary containing a connected component $M \subset \partial W$ that satisfies Weak Property ( $\star$ ). Then $\partial W$ is connected.

Theorem 2.9 Let $(W, \omega)$ be a compact and connected 4-manifold with convex boundary $(M, \xi)$ satisfying Weak Property $(\star)$. Then any closed contact type hypersurface $H$ in $W \backslash M$ separates $W$ into a convex filling of $H$ and a symplectic cobordism from $H$ to $M$. In particular, $H$ also satisfies Weak Property ( $\star$ ).

Remark 2.10 A compact connected symplectic manifold with convex boundary can never contain a symplectic sphere of nonnegative self-intersection. This follows easily 
from the arguments we will use to prove the above results: otherwise one would find a family of embedded holomorphic spheres foliating the positive end of the symplectization of the convex boundary, and thus violating the maximum principle.

Remark 2.11 Note that Property $(\star)$ depends only on the contact structure: we do not assume in any of these theorems that the contact form induced on $M$ by a Liouville vector field is the same one which appears in Definition 2.5.

We will show in Section 3 that any contact manifold $(M, \xi)$ with Giroux torsion satisfies Property $(\star)$. It turns out that this is also true for a contact fiber sum of open books $(M, \xi)=\#_{\Phi}\left(M_{i}, \xi_{i}\right)$ whenever any of the summands $\left(M_{i}, \xi_{i}\right)$ is planar. This follows from an important relationship between open books and holomorphic curves: namely, it is shown in both Abbas and Wendl $[1 ; 38]$ that if the open book on $\left(M_{i}, \xi_{i}\right)$ is planar, one can take its pages to be projected images of embedded index 2 holomorphic curves. A variation on this construction in Wendl [37] extends it to the blown up manifold $\left(\widehat{M}_{i}, \widehat{\xi}_{i}\right)$ : the difference here is that each holomorphic page is asymptotic to a different orbit in a Morse-Bott family foliating the boundary. Moreover, one can arrange the contact form in this construction so that all the asymptotic orbits are either elliptic or Morse-Bott and have much smaller period than any other Reeb orbit in $\#_{\Phi}\left(M_{i}, \xi_{i}\right)$. It follows that $\#_{\Phi}\left(M_{i}, \xi_{i}\right)$ satisfies Property $(\star)$ if any of its constituent open books is planar.

\section{Giroux torsion}

Following a construction of Wendl in [40], but taking greater care over the size of periods, we now establish the following proposition.

Proposition 3.1 Let $(M, \xi)$ be a closed contact manifold having Giroux torsion. Then $(M, \xi)$ satisfies Property $(\star)$.

Proof By definition, Giroux torsion means that $(M, \xi)$ contains a subset $T$ that can be identified with a thickened torus $S^{1} \times S^{1} \times[0,1]$, on which $\xi$ has the form

$$
\xi=\operatorname{ker}(\cos (2 \pi \theta) d x+\sin (2 \pi \theta) d y)
$$

in coordinates $(x, y, \theta) \in S^{1} \times S^{1} \times[0,1]$. Let us assume $\xi=\operatorname{ker} \lambda$ for some contact form $\lambda$ that is Morse-Bott outside of $T$, and in $T$ has the form $\lambda=f(\theta) d x+g(\theta) d y$ for smooth functions $f, g:[0,1] \rightarrow \mathbb{R}$ with

$$
\gamma(\theta):=(f(\theta), g(\theta))=h(\theta) e^{2 \pi i \theta} \in \mathbb{R}^{2},
$$


where $h(\theta)>0$ and $h(\theta)=1$ for $\theta$ near 0 and 1 . The path $\gamma$ is thus closed and bounds a star-shaped region in $\mathbb{R}^{2}$, and we will show that $\lambda$ has the desired properties if $\gamma$ bounds a suitably oblong oval.

The Reeb vector field of $\lambda$ on $T$ is given by

$$
X_{\lambda}=\frac{1}{D(\theta)}\left(g^{\prime}(\theta) \partial_{x}-f^{\prime}(\theta) \partial_{y}\right),
$$

where $D(\theta):=f(\theta) g^{\prime}(\theta)-f^{\prime}(\theta) g(\theta)>0$. Since this has no $\partial_{\theta}$ component, each torus $N\left(\theta_{0}\right):=\left\{\left(x, y, \theta_{0}\right) \mid(x, y) \in S^{1} \times S^{1}\right\} \subset T$ is invariant under the Reeb flow. Moreover, the Reeb flow on each $N(\theta)$ is linear and has closed orbits if and only if $d x\left(X_{\lambda}\right) / d y\left(X_{\lambda}\right) \in \mathbb{Q} \cup\{\infty\}$. From (3-2), this ratio is $-g^{\prime}(\theta) / f^{\prime}(\theta)=-\operatorname{slope}\left(\gamma^{\prime}(\theta)\right)$, so $N(\theta)$ has closed orbits precisely when slope $\left(\gamma^{\prime}(\theta)\right)$ is rational or infinite. In this case every orbit in $N(\theta)$ is closed and represents the same class in $H_{1}(N(\theta))=\mathbb{Z}^{2}$, which we will denote by a pair of integers $(p(\theta), q(\theta))$ with $\operatorname{gcd}(|p(\theta)|,|q(\theta)|)=1$ and

$$
\frac{p(\theta)}{q(\theta)}=-\operatorname{slope}\left(\gamma^{\prime}(\theta)\right) \in \mathbb{Q} \cup\{\infty\} .
$$

Since $d \lambda$ vanishes on $N(\theta)$, all closed simply covered orbits in $N(\theta)$ have the same period, which we will denote by $\mathcal{T}(\theta)>0$. If $\sigma: \mathbb{R} / \mathbb{Z} \rightarrow N(\theta)$ parametrizes such an orbit, we compute

$$
\mathcal{T}(\theta)=\int_{0}^{1} \sigma^{*} \lambda=p(\theta) f(\theta)+q(\theta) g(\theta) .
$$

Lemma 3.2 Fix $\epsilon>0$ small and assume that in addition to the above conditions, $\gamma(\theta)=h(\theta) e^{2 \pi i \theta}$ bounds a convex set symmetric about both axes, $h(1 / 4)=h(3 / 4)=$ $\epsilon$ and $\gamma^{\prime}(\theta)$ and $\gamma^{\prime \prime}(\theta)$ are always linearly independent. Then:

(1) $\lambda$ is Morse-Bott.

(2) $X_{\lambda}=(1 / \epsilon) \partial_{y}$ on $N(1 / 4)$ and $-(1 / \epsilon) \partial_{y}$ on $N(3 / 4)$.

(3) $\mathcal{T}(1 / 4)=\mathcal{T}(3 / 4)=\epsilon$, and $\mathcal{T}(\theta)>1 / 4$ for all other $\theta$ at which $N(\theta)$ has closed orbits.

Proof It follows by straightforward computation from the assumption that $\gamma^{\prime}(\theta)$ and $\gamma^{\prime \prime}(\theta)$ are linearly independent that each $N(\theta)$ with closed orbits is a MorseBott submanifold. The second claim follows immediately from (3-2) since symmetry requires $g^{\prime}(1 / 4)=g^{\prime}(3 / 4)=0$, and it is then clear that $\mathcal{T}(1 / 4)=\mathcal{T}(3 / 4)=\epsilon$.

To show that all other values of $\theta$ have $\mathcal{T}(\theta)>1 / 4$, observe first that by symmetry, we can always assume $g^{\prime}$ and $-f^{\prime}$ have the same sign as $f$ and $g$ respectively. 
Thus $\operatorname{sign}(p)=\operatorname{sign}\left(d x\left(X_{\lambda}\right)\right)=\operatorname{sign}\left(g^{\prime}\right)=\operatorname{sign}(f)$ and $\operatorname{sign}(q)=\operatorname{sign}\left(d y\left(X_{\lambda}\right)\right)=$ $\operatorname{sign}\left(-f^{\prime}\right)=\operatorname{sign}(g)$, so formula (3-4) becomes

$$
\mathcal{T}(\theta)=|p(\theta)||f(\theta)|+|q(\theta)||g(\theta)| .
$$

Let $\Delta$ denote the diamond shaped region in the $x y$-plane for which $|x|+|y| \leq 1 / 2$ (see Figure 3). We deal separately with two cases.

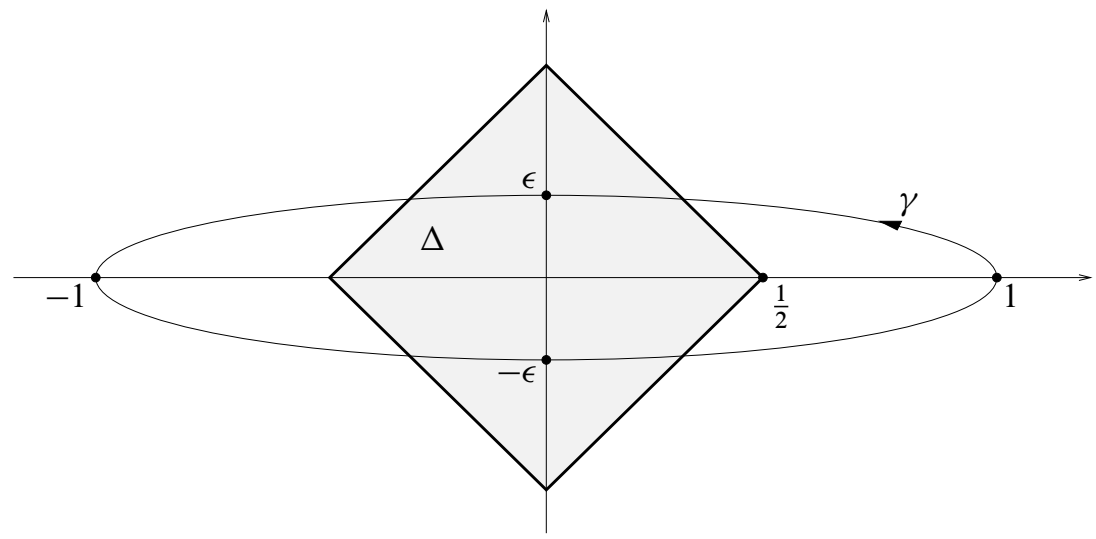

Figure 3: The curve $\gamma$ and (shaded) region $\Delta$ in Lemma 3.2

Case $\gamma(\theta) \in \Delta \quad$ In this region, outside of the special values $\theta=1 / 4,3 / 4$ we have $0<\left|\operatorname{slope}\left(\gamma^{\prime}(\theta)\right)\right|<2 \epsilon$, and by convexity, $|g(\theta)|>\epsilon / 2$. With the slope nonzero, it follows from (3-3) that both $p$ and $q$ are nonzero: in particular $|p| \geq 1$. Then from the previous inequality

$$
|q|=\frac{|q|}{|p|}|p|=\frac{1}{\left|\operatorname{slope}\left(\gamma^{\prime}(\theta)\right)\right|}|p|>\frac{1}{2 \epsilon}|p| \geq \frac{1}{2 \epsilon},
$$

and using (3-5), we obtain $\mathcal{T}(\theta) \geq|q(\theta)||g(\theta)|>(1 /(2 \epsilon))(\epsilon / 2)=1 / 4$.

Case $\gamma(\theta) \notin \Delta$ After verifying explicitly that $\mathcal{T}(0)=\mathcal{T}(1)=1$, we can exclude these two cases and assume once more that both $p(\theta)$ and $q(\theta)$ are nonzero. Then (3-5) gives

$$
\mathcal{T}(\theta) \geq|f(\theta)|+|g(\theta)|>1 / 2
$$

by the definition of $\Delta$.

Using the lemma, we can arrange $\lambda$ in $T$ without changing it in $M \backslash T$ so that $\mathcal{T}(1 / 4)=\mathcal{T}(3 / 4)=\epsilon$ is less than half the period of every other periodic orbit in $M$. Now copying the original construction in Wendl [40, Example 2.11], we construct a 
family of embedded $J$-holomorphic cylinders in $\mathbb{R} \times T$ that foliate the region between $N(1 / 4)$ and $N(3 / 4)$, each of the form

$$
u: \mathbb{R} \times S^{1} \rightarrow \mathbb{R} \times M:(s, t) \mapsto\left(\alpha(s)+a_{0}, x_{0}, t, \rho(s)\right),
$$

where $a_{0} \in \mathbb{R}$ and $x_{0} \in S^{1}$ are arbitrary constants, $\alpha: \mathbb{R} \rightarrow \mathbb{R}$ is a fixed function that goes to $+\infty$ at both ends and $\rho: \mathbb{R} \rightarrow(1 / 4,3 / 4)$ is a fixed orientation reversing diffeomorphism. Any of these cylinders satisfies the requirements of Property $(\star)$.

\section{Fredholm theory, intersection numbers and compactness}

In this section, assume $(W, \omega)$ is a connected (and possibly noncompact) symplectic 4-manifold with convex boundary $\partial W=M$. The boundary need not be connected or nonempty; for simplicity we will assume that it is compact, though we will later be able to relax this assumption. Choosing a Liouville vector field $Y$ and a smooth function $f: M \rightarrow \mathbb{R}$, we define a contact form $\lambda$ on $M$ by $\left.\iota_{Y} \omega\right|_{M}=e^{f} \lambda$ and denote by $\xi=\operatorname{ker} \lambda$ the induced contact structure. We can then use the reverse flow of $Y$ to identify a neighborhood of $\partial W$ symplectically with a neighborhood of the boundary of $\left(\{(t, m) \in \mathbb{R} \times M \mid t \leq f(m)\}, d\left(e^{t} \lambda\right)\right)$. Thus we can smoothly attach the cylindrical end

$$
E_{+}:=(\{(t, m) \in \mathbb{R} \times M \mid t \geq f(m)\}
$$

with symplectic form $d\left(e^{t} \lambda\right)$, forming an enlarged symplectic manifold $\left(W^{\infty}, \omega\right)$ which naturally contains $\left([T, \infty) \times M, d\left(e^{t} \lambda\right)\right)$ for sufficiently large $T$.

Assumption 4.1 With $(W, \omega)$ as described above, assume either of the following:

(1) $(W, \omega)$ contains a symplectically embedded sphere $u_{0}: S^{2} \rightarrow W$ with selfintersection number zero.

(2) $(M, \xi)$ satisfies Property $(\star)$.

In the first case, we can define $\dot{\Sigma}:=S^{2}$ with the standard complex structure, choose any admissible $\mathbb{R}$-invariant almost complex structure $J_{+}$on $\left([T, \infty) \times M, d\left(e^{t} \lambda\right)\right)$ and extend it to an $\omega$-compatible almost complex structure $J$ on $W^{\infty}$ such that $u_{0}$ is (after reparametrization) a $J$-holomorphic curve. In the second case, we can (by appropriate choice of the function $f$ ) take $\lambda$ and $J_{+}$to be the particular contact form and almost complex structure arising from Definition 2.5, and again extend $J_{+}$to an $\omega$-compatible structure $J$ on $W^{\infty}$. After a sufficiently large $\mathbb{R}$-translation, the $J_{+}-$ holomorphic curve given by Definition 2.5 may then be regarded as a $J$-holomorphic curve

$$
u_{0}=\left(u_{\mathbb{R}}, u_{M}\right): \dot{\Sigma} \rightarrow[T, \infty) \times M \subset W^{\infty},
$$

where $\dot{\Sigma}=S^{2} \backslash\left\{z_{1}, \ldots, z_{N}\right\}$ with the standard complex structure of $S^{2}$. 
Given any smooth function $\varphi: \mathbb{R} \rightarrow(0, \infty)$ that is monotone increasing and satisfies $\varphi(t)=e^{t}$ for $t \leq T$, we can define a new symplectic form on $W^{\infty}$ by

$$
\omega_{\varphi}= \begin{cases}\omega & \text { in } W, \\ d(\varphi \lambda) & \text { in } E_{+} .\end{cases}
$$

Observe that $J$ is also compatible with $\omega_{\varphi}$.

Definition 4.2 The energy of a $J$-holomorphic curve $u$ : $\dot{\Sigma} \rightarrow W^{\infty}$ is

$$
E(u)=\sup _{\varphi \in \mathcal{T}} \int_{\dot{\Sigma}} u^{*} \omega_{\varphi},
$$

where $\omega_{\varphi}$ is as defined in (4-1) and $\mathcal{T}$ is the set of all smooth functions $\varphi: \mathbb{R} \rightarrow(0, \infty)$ that satisfy $\varphi^{\prime}>0, \varphi(t)=e^{t}$ for $t \leq T$ and $\sup \varphi \leq e^{2 T}$.

This is equivalent to the definition of energy given by Bourgeois-Eliashberg-Hofer [3], in the sense that uniform bounds on either imply uniform bounds on the other. As in Section 2.1, we will always assume that finite energy $J$-holomorphic curves in $W^{\infty}$ are proper and thus have no removable punctures: then they also satisfy the asymptotic formula (2-4) and thus have well defined asymptotic eigenfunctions and transversal convergence rates at each puncture.

Denote by $\mathcal{M}^{*}$ the moduli space of all proper, somewhere injective finite energy $J$-holomorphic curves in $W^{\infty}$, with arbitrary conformal structures on the domains and any two curves considered equivalent if they are related by a biholomorphic reparametrization that preserves each puncture. We assign to $\mathcal{M}^{*}$ the natural topology defined by $C^{\infty}$-convergence on compact subsets and $C^{0}$-convergence up to the ends, and denote by $\mathcal{M}_{0}^{*} \subset \mathcal{M}^{*}$ the connected component containing $u_{0}$. Observe that since $\int u^{*} \omega_{\varphi}$ depends only on $\varphi$ and the relative homology class represented by $u$, the energy $E(u)$ is uniformly bounded for all $u \in \mathcal{M}_{0}^{*}$.

We shall now define special subsets $\mathcal{M}^{\mathbf{c}} \subset \mathcal{M}^{*}$ and $\mathcal{M}_{0}^{\mathrm{c}} \subset \mathcal{M}_{0}^{*}$, consisting of $J-$ holomorphic curves that satisfy asymptotic constraints. If $u_{0}$ has no punctures, we can simply set $\mathcal{M}^{\mathbf{c}}=\mathcal{M}^{*}$ and $\mathcal{M}_{0}^{\mathbf{c}}=\mathcal{M}_{0}^{*}$. Otherwise, let us fix the following notation: for each puncture $z \in \Gamma$ of $u_{0}$, denote the corresponding asymptotic orbit of $u_{0}$ by $\gamma_{z}$, with asymptotic operator $\mathbf{A}_{z}$, asymptotic eigenfunction $e_{z}$ and transversal convergence rate $-\mu_{z}$, so $\mu_{z} \in \sigma\left(\mathbf{A}_{z}\right)$. Choose any unitary trivialization $\Phi$ for $\xi$ along each of the orbits $\gamma_{z}$. We will define a new partition

$$
\Gamma=\Gamma_{C} \cup \Gamma_{U}
$$


in terms of the asymptotic behavior of $u_{0}$, calling these the constrained and unconstrained punctures respectively. Namely, define $z \in \Gamma$ to be in $\Gamma_{C}$ if and only if $\gamma_{z}$ is either nondegenerate or belongs to a Morse-Bott submanifold $N \subset M$ that intersects $u_{M}(\dot{\Sigma})$.

Lemma 4.3 If $\gamma_{z}$ belongs to a Morse-Bott submanifold $N \subset M$ of dimension at least 2, then $N$ intersects $u_{M}(\dot{\Sigma})$ if and only if wind ${ }^{\Phi}\left(e_{z}\right)<0$, where $\Phi$ is the unique trivialization in which the nontrivial sections in $\operatorname{ker} \mathbf{A}_{z}$ have zero winding.

Proof It is obvious from the asymptotic formula (2-4) that $u_{M}$ intersects $N$ if wind $^{\Phi}\left(e_{z}\right)<0$. To prove the converse, observe first that since $u_{M}$ is embedded, it cannot intersect its own asymptotic orbits. One then has to show that if $u_{0}$ intersects any trivial cylinder $\mathbb{R} \times \gamma^{\prime}$ over an orbit $\gamma^{\prime}$ in $N$, then it also has an "asymptotic intersection" with $\mathbb{R} \times \gamma_{z}$, which cannot be true if wind ${ }^{\Phi}\left(e_{z}\right)=0$. This follows easily from the intersection theory of punctured holomorphic curves; see Siefring [31] and also Siefring-Wendl [32] for details.

Lemma 4.4 For each $z \in \Gamma_{C}$, there exists a number $c_{z}<0$ such that $c_{z} \notin \sigma\left(\mathbf{A}_{z}\right)$, $\alpha_{-}^{\Phi}\left(\gamma_{z}-c_{z}\right)=\operatorname{wind}^{\Phi}\left(e_{z}\right)$ and $\alpha_{+}^{\Phi}\left(\gamma_{z}-c_{z}\right)=\operatorname{wind}^{\Phi}\left(e_{z}\right)+1$.

Proof Choose $\Phi$ so that wind ${ }^{\Phi}\left(e_{z}\right)=0$; in the language of Definition 2.5, this is the special trivialization determined by the asymptotic behavior of $u_{M}$ near $z$. Then $\alpha_{-}^{\Phi}\left(\gamma_{z}\right) \geq 0$, and if $\gamma_{z}$ is nondegenerate, (2-2) implies $\mu_{\mathrm{CZ}}^{\Phi}\left(\gamma_{z}\right) \geq 0$, with equality if and only if $\alpha_{-}^{\Phi}\left(\gamma_{z}\right)=\alpha_{+}^{\Phi}\left(\gamma_{z}\right)=0$. The latter is therefore excluded by the condition $\mu_{\mathrm{CZ}}^{\Phi}\left(\gamma_{z}\right) \neq 0$ from Definition 2.5. It follows that if $\mu \in \sigma\left(\mathbf{A}_{z}\right)$ is the largest eigenvalue with wind ${ }^{\Phi}(\mu)=$ wind $^{\Phi}\left(e_{z}\right)$, then $\mu<0$ and we can choose $c_{z}$ to be any number slightly larger than $\mu$.

For the case where $\gamma_{z}$ is Morse-Bott, the fact that $u_{M}$ intersects the Morse-Bott submanifold means $0=$ wind $^{\Phi}\left(e_{z}\right)<$ wind $^{\Phi}(0)$ due to Lemma 4.3. Thus the eigenvalue $\mu$ defined above is again negative and we can choose $c_{z}$ to be slightly larger.

In the following, let $c_{z}<0$ denote the number given by Lemma 4.4 for each constrained puncture $z \in \Gamma_{C}$, and for $z \in \Gamma_{U}$ set $c_{z}:=\epsilon>0$ small enough so that $(0, \epsilon)$ never intersects $\sigma\left(\mathbf{A}_{z}\right)$.

Definition 4.5 The constrained moduli space $\mathcal{M}^{\mathbf{c}}$ consists of all curves $u \in \mathcal{M}^{*}$ having at most \#Г punctures, which can be identified with a subset of $\Gamma$ in such a way that at every $z \in \Gamma_{C}$ that is a puncture of $u$, the asymptotic orbit of $u$ is $\gamma_{z}$, with transversal convergence rate strictly greater than $\left|c_{z}\right|$. Let $\mathcal{M}_{0}^{\mathbf{c}} \subset \mathcal{M}^{\mathbf{c}}$ denote the connected component containing $u_{0}$. 
Proposition 4.6 Every curve $u \in \mathcal{M}_{0}^{\mathbf{c}}$ is embedded.

Proof By Definition 2.5, each asymptotic orbit for the curves in $\mathcal{M}_{0}^{\mathbf{c}}$ is either fixed or allowed to vary in a Morse-Bott torus that contains no other asymptotic orbits, thus the orbits of each $u \in \mathcal{M}_{0}^{\mathrm{c}}$ are all distinct and simply covered. It follows that embedded curves form an open subset of $\mathcal{M}_{0}^{\mathbf{c}}$, which is also nonempty since it contains $u_{0}$. By positivity of intersections, it is also closed, so the claim follows from the assumption that $\mathcal{M}_{0}^{\mathbf{c}}$ is connected.

Topologically, $\mathcal{M}^{\mathbf{c}}$ is a closed subspace of $\mathcal{M}^{*}$. Recall that $\mathcal{M}^{*}$ can locally be identified (up to symmetries) with the zero set of the nonlinear Cauchy-Riemann operator $\bar{\partial}_{J}$, regarded as a smooth section of a certain Banach space bundle. The same is true for $\mathcal{M}^{\mathbf{c}}$, but with Banach spaces of maps whose behavior at the ends satisfies exponential weighting constraints determined by the numbers $c_{z}$. We refer to [34; 39 ] for details on the general analytical setup, and $[22 ; 35 ; 39]$ for the exponential weights. A given curve $u \in \mathcal{M}^{\mathbf{c}}$ is called Fredholm regular if the linearization of $\bar{\partial}_{J}$ at $u$ is surjective. In general, this linearization is a Fredholm operator, whose index (with correction terms for the dimensions of Teichmüller space and the automorphism group) defines the "virtual dimension" of the moduli space near $u$. We'll denote this virtual dimension by $\operatorname{ind}(u ; \mathbf{c})$, and call it the (constrained) index of $u$. If $u$ is Fredholm regular, then the implicit function theorem implies that $\mathcal{M}^{\mathbf{c}}$ near $u$ is a smooth manifold, whose dimension is given by the index.

Theorem 4.7 Every $u \in \mathcal{M}_{0}^{\mathbf{c}}$ is Fredholm regular and has $\operatorname{ind}(u ; \mathbf{c})=2$. Moreover, a neighborhood of $u$ in $\mathcal{M}_{0}^{\mathbf{c}}$ forms a smooth 2-parameter family $\left\{u^{\tau}\right\}_{\tau \in \mathbb{D}}$, with $u^{0}=u$, such that:

(1) The images $u^{\tau}(\dot{\Sigma})$ foliate a neighborhood of $u(\dot{\Sigma})$ in $W$.

(2) For any puncture $z \in \Gamma_{U}$, the set of all curves $\left\{u^{\tau}\right\}_{\tau \in \mathbb{D}}$ that approach the same orbit as $u$ at $z$ is a smooth 1-dimensional submanifold.

Proof We first verify the claim that $\operatorname{ind}(u ; \mathbf{c})=2$. For the case where $u$ is a closed embedded sphere with self-intersection zero, this follows immediately from the adjunction formula: $0=u \bullet u=c_{1}\left(u^{*} T W^{\infty}\right)-2$, thus $c_{1}\left(u^{*} T W^{\infty}\right)=2$ and $\operatorname{ind}(u)=-2+2 c_{1}\left(u^{*} T W^{\infty}\right)=2$.

In the case where $u_{0}$ arises from Property $(\star)$, it suffices to prove that $\operatorname{ind}\left(u_{0} ; \mathbf{c}\right)=2$ with $u_{0}$ regarded as a $J_{+}$-holomorphic curve in $\mathbb{R} \times M$. Recall from [35] that one can associate with $u_{0}$ an integer $c_{N}\left(u_{0} ; \mathbf{c}\right)$, called the (constrained) normal Chern number, which satisfies

$$
2 c_{N}\left(u_{0} ; \mathbf{c}\right)=\operatorname{ind}\left(u_{0} ; \mathbf{c}\right)-2+2 g+\# \Gamma_{0}(\mathbf{c}),
$$


where $g$ is the genus of $\dot{\Sigma}$ (in this case zero) and $\Gamma_{0}(\mathbf{c})$ is the subset of punctures $z \in \Gamma$ at which $p\left(\gamma_{z}-c_{z}\right)=0$. It also satisfies

$$
c_{N}\left(u_{0} ; \mathbf{c}\right)=\operatorname{wind}_{\pi}\left(u_{0}\right)+\sum_{z \in \Gamma}\left(\alpha_{-}^{\Phi}\left(\gamma_{z}-c_{z}\right)-\operatorname{wind}^{\Phi}\left(e_{z}\right)\right) .
$$

By Lemma 4.4 and the fact that $u_{M}: \dot{\Sigma} \rightarrow M$ is an embedding, the right hand side of (4-3) vanishes, implying $c_{N}\left(u_{0} ; \mathbf{c}\right)=0$. We claim also that $\# \Gamma_{0}(\mathbf{c})=0$, ie all punctures satisfy $p\left(\gamma_{z}-c_{z}\right)=1$; for $z \in \Gamma_{C}$ this already follows from Lemma 4.4. For unconstrained punctures $z \in \Gamma_{U}$, Lemma 4.3 implies that $e_{z}$ has the same winding number as a nontrivial section in $\operatorname{ker} \mathbf{A}_{z}$ : these also span the two eigenspaces of $\mathbf{A}_{z}-c_{z}=\mathbf{A}_{z}-\epsilon$ with negative eigenvalues closest to zero. It follows that every positive eigenvalue of $\mathbf{A}_{z}-\epsilon$ has strictly larger winding, thus $p\left(\gamma_{z}-\epsilon\right)=1$ as claimed. Now (4-2) implies ind $\left(u_{0} ; \mathbf{c}\right)=2$.

The remainder of the proof consists of minor generalizations of well established results from [22; 41], so we shall merely sketch the main ideas. Since $u \in \mathcal{M}_{0}^{\mathbf{c}}$ is embedded, the regularity question can be reduced to the study of the normal Cauchy-Riemann operator $\mathbf{D}_{u}^{N}$ as in $[18 ; 22 ; 34]$. The domain of $\mathbf{D}_{u}^{N}$ is an exponentially weighted Banach space of sections of the normal bundle $N_{u} \rightarrow \dot{\Sigma}$, and the sections in $\operatorname{ker} \mathbf{D}_{u}^{N}$ have only positive zeroes, whose algebraic count is bounded in general by $c_{N}(u ; \mathbf{c})$; $\operatorname{cf}$ [34]. In our case $c_{N}(u ; \mathbf{c})=c_{N}\left(u_{0} ; \mathbf{c}\right)=0$, thus every section in $\operatorname{ker} \mathbf{D}_{u}^{N}$ is zero free; a simple linear independence argument then shows that $\operatorname{dim} \operatorname{ker} \mathbf{D}_{u}^{N} \leq 2=\operatorname{ind} \mathbf{D}_{u}^{N}$, hence $\mathbf{D}_{u}^{N}$ is surjective. This shows that $\mathcal{M}_{0}^{\mathbf{c}}$ is a smooth 2-manifold near $u$, and $T_{u} \mathcal{M}_{0}^{\mathbf{c}}$ is identified with a space of smooth nowhere vanishing sections $\operatorname{ker} \mathbf{D}_{u}^{N} \subset \Gamma\left(N_{u}\right)$, implying the claim that the curves near $u$ foliate a neighborhood.

Finally we note that for each $z \in \Gamma_{U}$, one can apply an additional constraint to study subspaces of curves in $\mathcal{M}_{0}^{\mathrm{c}}$ that fix the position of the asymptotic orbit. In the linearization this amounts to replacing $c_{z}=\epsilon$ by $c_{z}=-\epsilon$; this idea is explained in detail in Wendl $[41 ; 39]$. The problem with the additional constraint then has index 1 and is again regular by an argument using the formal adjoint of $\mathbf{D}_{u}^{N}$, as in [34].

Note that in the above proof, Fredholm regularity does not require any genericity assumptions, rather it comes for free due to "automatic" transversality (cf [34]). As a consequence, $u_{0}$ can be deformed with sufficiently small perturbations of $J$ and $\lambda$ so that Theorem 4.7 still applies. After such a perturbation (using Lemma 2.2), we can therefore assume the following from now on:

(1) All orbits of period less than some large constant $C>0$ are Morse-Bott.

(2) $J$ is generic outside of $[T, \infty) \times M$, so that in particular every curve $u \in \mathcal{M}^{\mathrm{c}}$ that isn't wholly contained in $[T, \infty) \times M$ has $\operatorname{ind}(u ; \mathbf{c}) \geq 0$. 
The exact details of our generic perturbation of $J$ are somewhat delicate and specific to the application we have in mind; this will be explained in Lemma 5.2 in Section 5. Note that the purpose of this assumption has nothing to do with the curves in $\mathcal{M}_{0}^{\mathbf{c}}$, which are already regular-rather we will see below that genericity is needed to gain control over the degenerations that can occur in the natural compactification of $\mathcal{M}_{0}^{\mathbf{c}}$.

Due to the Morse-Bott assumption, the compactness theorem of [3] now applies to any sequence of $J$-holomorphic curves in $W^{\infty}$ that satisfy a suitable $C^{0}$-bound and energy bound: in particular, such a sequence has a subsequence that converges to a nodal holomorphic building, typically with multiple levels. In our situation, the bottom level will be a nodal $J$-holomorphic curve in $W^{\infty}$, and all levels above this are nodal $J_{+}$-holomorphic curves in $\mathbb{R} \times M$.

Theorem 4.8 Suppose $u_{k} \in \mathcal{M}_{0}^{\mathbf{c}}$ is a sequence whose images are all contained in $W_{0} \cup E_{+}$for some compact subset $W_{0} \subset W$. Then a subsequence of $u_{k}$ converges to one of the following:

(1) another smooth curve in $\mathcal{M}_{0}^{\mathbf{c}}$,

(2) a holomorphic building with empty bottom level and one nontrivial upper level that consists of a smooth, embedded $J_{+}$-holomorphic curve in $\mathbb{R} \times M$ satisfying the conditions of Property $(\star)$,

(3) a nodal $J$-holomorphic curve in $W^{\infty}$ with exactly two components, both in $\mathcal{M}^{\mathbf{c}}$ and both embedded with (constrained) index 0 .

Moreover the set of index 0 curves that can appear as components of nodal curves in the third case is finite.

Before we prove the theorem we state the following important corollary. For this, we denote by $S \subset W^{\infty}$ the set through which the finitely many limit curves from part (3) of Theorem 4.8 pass, and let $C \subset W^{\infty} \backslash S$ consist of all points that are contained in curves from $\mathcal{M}_{0}^{\mathbf{c}}$.

Corollary 4.9 In addition to the assumptions of Theorem 4.8, assume that the images of all curves in $\mathcal{M}_{0}^{\mathbf{c}}$ are contained in $W_{0} \cup E_{+}$for some compact subset $W_{0} \subset W$. Then $C=W^{\infty} \backslash S$, and thus $W$ is compact.

Proof We claim that $C$ is a nonempty, open and closed subset of $W^{\infty} \backslash S$. It is clearly nonempty since $\mathcal{M}_{0}^{\mathbf{c}}$ also is, by construction. Openness is a direct consequence of Theorem 4.7 part (1). To prove that $C$ is closed, we choose a sequence $\left(p_{n}\right) \subset C$ with $p_{n} \rightarrow p^{*} \in W^{\infty} \backslash S$. Then by definition, there exist curves $u_{n} \in \mathcal{M}_{0}^{\mathbf{c}}$ with 
$p_{n} \in \operatorname{im}\left(u_{n}\right)$. A subsequence of $u_{n}$ converges to a holomorphic building $u^{*}$, which by Theorem 4.8 is either a smooth curve or a nodal curve with one level. Since $p^{*}$ is in the image of $u^{*}$ and $p^{*} \notin S$, we conclude that $u^{*} \in \mathcal{M}_{0}^{\mathrm{c}}$ and $p^{*} \in \operatorname{im} u^{*} \subset C$.

Now, since $S$ is a finite union of images of holomorphic curves, $W^{\infty} \backslash S$ is connected and it follows from the above claim that $C=W^{\infty} \backslash S$. Since by assumption $C \subset$ $W_{0} \cup E_{+}$, we conclude that $W$ is compact.

In proving Theorem 4.8, we will make use of a few concepts from the intersection theory of punctured holomorphic curves; this theory is developed in detail in the papers [31; 32], and the last section of [34] also contains a summary. Assume $v_{1}, v_{2} \in \mathcal{M}^{\mathbf{c}}$. Then there is an algebraic intersection number

$$
i\left(v_{1} ; \mathbf{c} \mid v_{2} ; \mathbf{c}\right) \in \mathbb{Z}
$$

which has the following properties:

(1) $i\left(v_{1} ; \mathbf{c} \mid v_{2} ; \mathbf{c}\right)$ is unchanged under continuous variations of $v_{1}$ and $v_{2}$ in $\mathcal{M}^{\mathbf{c}}$.

(2) If $v_{1}$ and $v_{2}$ are not both covers of the same somewhere injective curve, then

$$
i\left(v_{1} ; \mathbf{c} \mid v_{2} ; \mathbf{c}\right) \geq 0,
$$

and the inequality is strict if they intersect.

Unlike the usual homological intersection theory applied to closed holomorphic curves, the last statement is not an "if and only if": it is possible in general for $v_{1}$ and $v_{2}$ to be disjoint even if $i\left(v_{1} ; \mathbf{c} \mid v_{2} ; \mathbf{c}\right)>0$, though this phenomenon is in some sense nongeneric. The intersection number can also be defined for curves in the symplectization $\mathbb{R} \times M$, possibly with both positive and negative punctures. In this case one has invariance under $\mathbb{R}$-translation, so if $i\left(v_{1} ; \mathbf{c} \mid v_{2} ; \mathbf{c}\right)=0$ then the projected images of $v_{1}$ and $v_{2}$ in $M$ never intersect.

Lemma 4.10 $i\left(u_{0} ; \mathbf{c} \mid u_{0} ; \mathbf{c}\right)=0$.

Proof Since $u_{0}$ has only simply covered Reeb orbits and all of them are distinct, it satisfies the following somewhat simplified version of the adjunction formula from Siefring [31] and Siefring-Wendl [32]:

$$
i\left(u_{0} ; \mathbf{c} \mid u_{0} ; \mathbf{c}\right)=2 \delta\left(u_{0}\right)+c_{N}\left(u_{0} ; \mathbf{c}\right) .
$$

Here $\delta\left(u_{0}\right)$ is the algebraic count of double points and singularities of $u_{0}$ (see McDuff and Salamon [28]), which vanishes since $u_{0}$ is embedded. As we saw in the proof of Theorem 4.7, $c_{N}\left(u_{0} ; \mathbf{c}\right)$ also vanishes, so the claim follows. 
Lemma 4.11 If $v \in \mathcal{M}_{0}^{\mathrm{c}}$ is contained in $[T, \infty) \times M \subset W^{\infty}$, then its projection to $M$ is embedded.

Proof Write $v=\left(v_{\mathbb{R}}, v_{M}\right): \dot{\Sigma} \rightarrow[T, \infty) \times M$. By assumption, $v$ can be deformed continuously to $u_{0}$ through $\mathcal{M}^{\mathbf{c}}$, thus $i(v ; \mathbf{c} \mid v ; \mathbf{c})=i\left(u_{0} ; \mathbf{c} \mid u_{0} ; \mathbf{c}\right)=0$ by the previous lemma, and $c_{N}(v ; \mathbf{c})=c_{N}\left(u_{0} ; \mathbf{c}\right)=0$. Now (4-3) implies that $\operatorname{wind}_{\pi}(v)=0$, thus $v_{M}$ is immersed, and the vanishing self-intersection number implies that $v$ has no intersections with any of its $\mathbb{R}$-translations, so $v_{M}$ is also injective.

Proof of Theorem 4.8 By Bourgeois, Eliashberg and Hofer [3], $u_{k}$ has a subsequence converging to some holomorphic building, which we'll denote by $u$. Our first task is to show that unless $u$ is a 2 -level building with empty bottom level as described in Case (2), it can have no nontrivial upper levels. This is already clear in the case where $u$ is closed, as convexity prevents $u_{k}$ from venturing into the region $[T, \infty) \times M$ at all. Let us therefore assume that $u_{k}$ has punctures and that $u$ has nontrivial upper levels. If no component in these upper levels has any negative punctures, then there must be only one nontrivial level, which consists of one or more connected components $v_{1}, \ldots, v_{N}$ attached to each other by nodes. All of these components have punctures, since the symplectic form in $\mathbb{R} \times M$ is exact; moreover, the positive ends of each $v_{i}$ correspond to some subset of the positive ends of $u_{0}$, and since these are all simply covered and distinct, each $v_{i}$ is somewhere injective and satisfies the asymptotic constraints defined by c. Now (4-2) and (4-3) give

$$
0 \leq 2 \operatorname{wind}_{\pi}\left(v_{i}\right) \leq 2 c_{N}\left(v_{i} ; \mathbf{c}\right)=\operatorname{ind}\left(v_{i} ; \mathbf{c}\right)-2,
$$

hence $\operatorname{ind}\left(v_{i} ; \mathbf{c}\right) \geq 2$. Since $\operatorname{ind}\left(u_{0} ; \mathbf{c}\right)=2$ as well, we conclude that $u$ can have at most one connected component, with no nodes, ie it is a smooth $J_{+}$-holomorphic curve in $\mathbb{R} \times M$ with only positive punctures. Up to $\mathbb{R}$-translation, $u$ can therefore be identified with some smooth curve in $\mathcal{M}_{0}^{\mathbf{c}}$ whose image is contained in $[T, \infty) \times M$, and the projection into $M$ is embedded due to Lemma 4.11. It follows that this curve satisfies the conditions of Property $(\star)$.

Alternatively, suppose $u$ has nontrivial upper levels and the top level contains a $J_{+-}$ holomorphic curve $u_{+}$in $\mathbb{R} \times M$ which is not the trivial cylinder over an orbit and has both positive and negative punctures. Repeating the above argument about behavior at the positive ends, $u_{+}$is somewhere injective. Applying Stokes' theorem to $\int u_{+}^{*} d \lambda \geq 0$, the negative asymptotic orbits of $u_{+}$have total period bounded by the total period of the positive orbits, implying that all of the negative orbits belong to the same Morse-Bott manifolds as the orbits of $u_{0}$. We claim that after some $\mathbb{R}$-translation, $u_{+}$intersects $u_{0}$. This will imply a contradiction almost immediately, as positivity of intersections then 
gives an intersection of $u_{k}$ with some $\mathbb{R}$-translation of $u_{0}$ for sufficiently large $k$, contradicting Lemma 4.10 since $i\left(u_{k} ; \mathbf{c} \mid u_{0} ; \mathbf{c}\right)=i\left(u_{0} ; \mathbf{c} \mid u_{0} ; \mathbf{c}\right)=0$.

To prove the claim, it suffices to show that the projected images of $u_{+}$and $u_{0}$ in $M$ intersect each other. Suppose $\gamma$ is an asymptotic orbit of $u_{0}$ that lies in the same Morse-Bott submanifold $N \subset M$ as one of the negative asymptotic orbits $\gamma^{\prime}$ of $u_{+}$. Denote the corresponding asymptotic eigenfunctions by $e$ and $e^{\prime}$ respectively. We consider the following cases:

Case $1 N$ is a circle. Then $\gamma$ is nondegenerate and $\gamma^{\prime}$ is the $k$-fold cover of $\gamma$ for some $k \in \mathbb{N}$. Choose a trivialization $\Phi$ along $\gamma$ so that wind $^{\Phi}(e)=0$. By Lemma 4.4, $\mathbf{A}_{\gamma}$ has two eigenvalues (counting multiplicity) $\mu<0$ with wind $^{\Phi}(\mu)=0$. Then the $k$-fold covers of their eigenfunctions are eigenfunctions of $\mathbf{A}_{\gamma^{\prime}}$ with negative eigenvalues and zero winding, implying that every positive eigenvalue of $\mathbf{A}_{\gamma^{\prime}}$ has strictly positive winding. Thus wind ${ }^{\Phi}\left(e^{\prime}\right) \geq \alpha_{+}^{\Phi}\left(\gamma^{\prime}\right)>0$, forcing the projections of $u_{0}$ and $u_{+}$in $M$ to intersect each other near $N$.

Case $2 N$ is a torus disjoint from $u_{M}$. Now $\gamma^{\prime}$ can be deformed through a 1parameter family of orbits to a $k$-fold cover of $\gamma$ for some $k \in \mathbb{N}$. Choose a trivialization $\Phi$ along every simply covered orbit in $N$ so that sections in the $0-$ eigenspaces have zero winding. By Lemma 4.3, $\mathbf{A}_{\gamma}$ has an eigenvalue $\mu<0$ such that wind $^{\Phi}(e)=$ wind $^{\Phi}(\mu)=0$, and taking $k$-fold covers of eigenfunctions, we similarly find eigenfunctions of $\mathbf{A}_{\gamma^{\prime}}$ that have zero winding and eigenvalues $k \mu<0$ and 0 . This implies that wind ${ }^{\Phi}\left(e^{\prime}\right) \geq \alpha_{+}^{\Phi}\left(\gamma^{\prime}\right)>0$, which forces the projection of $u_{+}$in $M$ to intersect $N$, ie $u_{+}$intersects a trivial cylinder $\mathbb{R} \times \gamma_{1}$ for some orbit $\gamma_{1} \subset N$. Then by the homotopy invariance of the intersection number, $u_{+}$also intersects $\mathbb{R} \times \gamma$. This intersection is transverse unless it occurs at a point where $\pi_{\lambda} \circ T u_{+}=0$, but the similarity principle implies that there are finitely many such points (see Hofer, Wysocki and Zehnder [19]). Thus if necessary we can use Theorem 4.7 to perturb $u_{0}$ and thus move $\gamma$ to a nearby orbit, so that the intersection of $\mathbb{R} \times \gamma$ with $u_{+}$is transverse. This implies a transverse intersection of the projected image of $u_{+}$in $M$ with $\gamma$, and therefore an intersection of the projections of $u_{+}$and $u_{0}$ nearby.

Case $3 N$ is a Morse-Bott manifold intersecting $u_{M}$. The argument is similar to Case 2, only now we use the intersection of $u_{M}$ with $N$ to show that $u_{M}$ intersects $\gamma^{\prime}$ and thus also the projected image of $u_{+}$near $\gamma^{\prime}$.

We've shown now that $u$ cannot have any nontrivial upper level except in Case (2), so it must therefore be a 1 -level building in $W^{\infty}$, ie a nodal $J$-holomorphic curve. The deduction of Case (3) now proceeds almost exactly as in the proof of [40, Theorem 7]. To summarize, the connected components of $u$ are all either punctured curves with positive ends at distinct simply covered orbits (and thus somewhere injective), or closed 
curves (which must be nonconstant by an index argument). The latter could in general be multiple covers, but if $v$ is a $k$-fold branched cover of some closed somewhere injective curve $v_{0}$, then we find $\operatorname{ind}(v)=k \cdot \operatorname{ind}\left(v_{0}\right)+2(k-1)$. Due to our genericity assumption, all somewhere injective curves have index at least 0 , so we find that the total index of $u$ becomes more than 2 unless there is at most one node connecting two components, and in this case both components must be somewhere injective. The adjunction formula (4-4) can now be used to show that these two components, $v_{1}$ and $v_{2}$, are both embedded, satisfy $i\left(v_{i} ; \mathbf{c} \mid v_{i} ; \mathbf{c}\right)=-1, i\left(v_{i} ; \mathbf{c} \mid u_{0} ; \mathbf{c}\right)=0$ and $i\left(v_{1} ; \mathbf{c} \mid v_{2} ; \mathbf{c}\right)=1$; moreover, they are both Fredholm regular and have (constrained) index 0 .

There's one minor point to address which was irrelevant in [40]: if there are no punctures, we haven't ruled out the possibility that $u$ is a smooth multiple cover, ie $u=v \circ \varphi$ for some closed somewhere injective sphere $v$ and holomorphic branched cover $\varphi: S^{2} \rightarrow S^{2}$. Since $c_{1}\left(u^{*} T W^{\infty}\right)=2$, this is allowed numerically only if $c_{1}\left(v^{*} T W^{\infty}\right)=1$ and $\varphi$ has degree 2 . But then we get a simple contradiction using the adjunction formula: since $u \bullet u=0$, the same holds for $v$, thus

$$
0=v \bullet v=2 \delta(v)+c_{1}\left(v^{*} T W^{\infty}\right)-2=2 \delta(v)-1
$$

where $\delta(v)$ is the algebraic count of double points and singularities. The right hand side is odd; in particular it can never be zero.

It remains to show that the set of all index 0 curves arising from nodal degenerations of $u_{k}$ is finite. Indeed, suppose $v_{k}$ is a sequence of finite energy $J$-holomorphic curves in $W^{\infty}$ with uniform energy and $C^{0}$-bounds such that:

(1) The punctures of $v_{k}$ are identified with a subset of $\Gamma$ and satisfy the asymptotic constraints of Definition 4.5.

(2) $i\left(v_{k} ; \mathbf{c} \mid u_{0} ; \mathbf{c}\right)=0$.

(3) $\operatorname{ind}\left(v_{k} ; \mathbf{c}\right)=0$.

Then we claim that $v_{k}$ has a convergent subsequence. The argument is familiar: we rule out nontrivial upper levels exactly as before by showing that any nontrivial component $v_{+}$in such a level must intersect $u_{0}$. Thus the only remaining possible nonsmooth limit is a nodal curve in $W^{\infty}$, but the same index argument now implies that there is at most one component, thus no nodes, and the limit is somewhere injective. It follows that this set of curves is a compact smooth 0 -dimensional manifold, ie a finite set. 


\section{Proofs of the main results}

\subsection{Proofs of Theorem 1.15 and Theorem 2.7}

We consider a closed and connected symplectic 4-manifold $(W, \omega)$ which contains a closed contact type hypersurface $M$ such that $W \backslash M$ is connected. Under the assumptions of Theorems 1.15 or 2.7, we will construct from this a noncompact symplectic manifold with convex boundary to which Corollary 4.9 applies, giving a contradiction. The general idea of the construction is outlined in Figure 4.

To start with, we compactify $W \backslash M$ by adding to each end a copy of $M$, obtaining a compact and connected symplectic manifold $\left(W_{1}, \omega\right)$ with one convex boundary component $M^{+}$and an identical concave boundary component $M^{-}$. Inductively, we define the compact symplectic manifold $W_{n}$ by $W_{n}:=W_{n-1} \cup_{M^{-}=M^{+}} W_{1}$, denoting the symplectic form on $W_{n}$ again by $\omega$. Note that $W_{n-1}$ is a compact symplectic submanifold of $W_{n}$ in a natural way. Thus the set

$$
(\mathcal{W}, \omega):=\bigcup_{n \geq 1}\left(W_{n}, \omega\right)
$$

is a noncompact symplectic manifold with convex boundary $M$ corresponding to the convex boundary of $W_{1}$.

Assume that $W$ contains a symplectically embedded sphere $S \subset W$ with $S \bullet S=N \geq 0$. Since $\omega$ is exact on $M$, Stokes' theorem implies that $S$ cannot be contained entirely in $M$. We can thus blow up $W$ at $N$ distinct points in $S$ that are not in $M$, modifying both $W$ and $S$ so that $S \bullet S=0$ without loss of generality. Now we claim that $S$ can be "lifted" to a symplectic sphere $\widetilde{S}$ in $(\mathcal{W}, \omega)$ with $\widetilde{S} \bullet \widetilde{S}=0$. To see this, construct a symplectic infinite cover $(\widetilde{W}, \widetilde{\omega})$ of $(W, \omega)$ by gluing together a sequence of copies $\left\{\left(W_{1}^{j}, \omega\right)\right\}_{j \in \mathbb{Z}}$ of $\left(W_{1}, \omega\right)$, with the concave boundary of $W_{1}^{j}$ attached to the convex boundary of $W_{1}^{j+1}$ for each $j \in \mathbb{Z}$. Since the sphere is simply connected, $S$ has a lift $\widetilde{S} \subset \widetilde{W}$, and moreover, $(\widetilde{W}, \widetilde{\omega})$ naturally contains $(\mathcal{W}, \omega)$, which we may assume contains $\widetilde{S}$ without loss of generality.

Similarly, if $M$ with its induced contact structure satisfies Weak Property $(\star)$, then after attaching a symplectic cobordism to the convex boundary of $(\mathcal{W}, \omega)$, we may assume without loss of generality that either $(\mathcal{W}, \omega)$ contains a symplectic sphere of zero self-intersection (after blowing up) or Property $(\star)$ holds for $\partial \mathcal{W}$.

In either case, $(\mathcal{W}, \omega)$ now satisfies Assumption 4.1. As explained in Section 4, we can then attach to $\partial \mathcal{W}$ a cylindrical end $E^{+}$that contains $\left([T, \infty) \times M, d\left(e^{t} \lambda\right)\right)$ for sufficiently large $T \in \mathbb{R}$ and a suitable contact form $\lambda$, obtaining an enlarged 

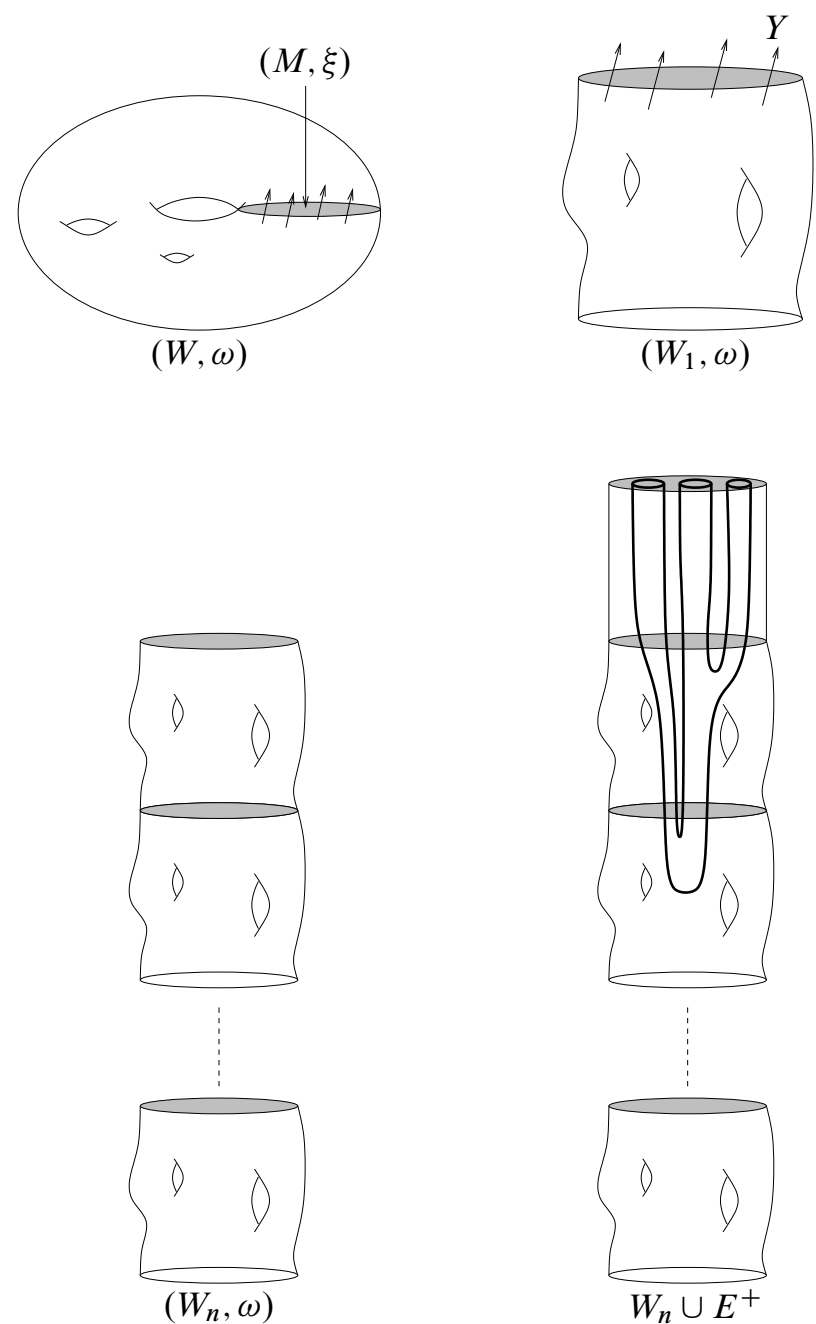

Figure 4: The compact symplectic manifold $(W, \omega)$ contains the nonseparating contact hypersurface $(M, \xi) . W \backslash M$ is compactified to produce $\left(W_{1}, \omega\right)$, which has two boundary components contactomorphic to $M$, one convex and one concave. Successively attaching $n$ copies of $W_{1}$ to itself produces $\left(W_{n}, \omega\right)$. Then Property $(\star)$ gives rise to a moduli space of finite energy curves which, due to the monotonicity lemma, cannot escape from $W_{n} \cup E^{+}$if $n$ is sufficiently large.

symplectic manifold $\left(\mathcal{W}^{\infty}, \omega\right)$, with an $\omega$-compatible almost complex structure $J_{0}$ that is admissible and $\mathbb{R}$-invariant on $[T, \infty) \times M$, and a nonempty moduli space $\mathcal{M}_{0}^{\mathbf{c}} \subset \mathcal{M}^{\mathbf{c}}$ of $J_{0}$-holomorphic curves in $\mathcal{W}^{\infty}$. Moreover for some $n_{0} \in \mathbb{N}$, we can assume that $J_{0}$ belongs to the following set. 
Definition 5.1 Let $\mathcal{J}^{\text {per }}$ be the space of compatible almost complex structures on $\left(\mathcal{W}^{\infty}, \omega\right)$ which match $J_{0}$ on $\left([T, \infty) \times M, d\left(e^{t} \lambda\right)\right)$ and whose restrictions to $W \cong$ $W_{n+1} \backslash W_{n} \subset \mathcal{W}^{\infty}$ are independent of $n$ for $n \geq n_{0}\left(J_{0}\right)$. Such a $J$ will be called periodic.

Lemma 5.2 For a generic $J \in \mathcal{J}^{\text {per }}$, all $J$-holomorphic curves in $\mathcal{M}^{\mathbf{c}}$ are Fredholm regular.

Proof Recall that the $J$-holomorphic curves in $\mathcal{M}^{\mathbf{c}}$ are somewhere injective; see Section 4. The proof of transversality is a small variation on the standard technique, as in McDuff and Salamon [28]: the key is to show that the universal moduli space $\{(u, J) \mid u$ is $J$-holomorphic $\}$ is a smooth Banach manifold for periodic $J$ and $u$ satisfying the relevant conditions. This will use the fact that a perturbation of $J$ can be localized at an injective point of $u$ without interfering at other points in the image of $u$. Then regular values of the projection $(u, J) \mapsto J$ are generic by the Sard-Smale theorem, and for these, all $J$-curves are Fredholm regular.

Assume $J \in \mathcal{J}^{\text {per }}$ and $u \in \mathcal{M}^{\mathbf{c}}$ is not fully contained in $[T, \infty) \times M$. If $u$ also intersects $W_{n_{0}} \cup E^{+}$, then it suffices to perturb $J$ only in this region and thus preserve periodicity of $J$. Thus it remains only to show that $\mathcal{J}^{\text {per }}$ permits sufficient perturbations of $J$ when the image of $u$ is contained in $\mathcal{W} \backslash W_{n_{0}}$, in which case $u$ must be a somewhere injective closed curve. Since $J$ is required to be periodic, the only danger not present in the standard case is that $u$ may have periodic points, in the following sense. Recall that $\mathcal{W}^{\infty}$ contains infinitely many identical copies of a certain manifold $V$, in the form $\widehat{W}_{n}:=W_{n+1} \backslash W_{n}$. Thus each point $x \in V$ appears infinitely often in $\mathcal{W}^{\infty}$, and we call these different points translates of $x$. Then $z \in \dot{\Sigma}$ is a periodic point of $u$ if a translate of $u(z)$ is contained in the image $\operatorname{im}(u)$ of $u$. In this case a periodic perturbation of $J$ cannot be localized in the image of $u$.

We claim that for any somewhere injective closed holomorphic curve in $\mathcal{W} \backslash W_{n_{0}}$, the set of injective points which are not periodic is open and dense. To see this, we can consider the covering space $\pi: \widetilde{W} \rightarrow W$ which was constructed above Definition 5.1. Since $J$ is periodic, the projection $\pi \circ u$ is a holomorphic curve in $W$. It will suffice to show that also $\pi \circ u$ is somewhere injective, since then the set of injective points of $\pi \circ u$ is open and dense, and injective points of $\pi \circ u$ give rise to nonperiodic injective points of $u$. Denote by $\tau: \widetilde{W} \rightarrow \widetilde{W}$ the deck transformation that maps $\widetilde{W}_{n}$ to $\widetilde{W}_{n+1}$. Then if $\pi \circ u$ is multiply covered, the fact that $u$ is somewhere injective implies (using unique continuation) that $u$ and $\tau^{k} \circ u$ are equivalent curves for some integer $k \neq 0$. But then $u$ is also equivalent to $\tau^{n k} \circ u$ for any $n \in \mathbb{Z}$, implying that the image of $u$ in $\widetilde{W}$ is unbounded. Since $u$ was assumed to be closed, this is a contradiction and shows that $\pi \circ u$ is indeed somewhere injective. 
With this, the usual proof that the universal moduli space is a smooth Banach manifold goes through unchanged.

For the remainder of this section we assume that the almost complex structure $J$ (formerly called $J_{0}$ ) is periodic and generic.

Proposition 5.3 There exists $N_{0} \in \mathbb{N}$ such that for all $u \in \mathcal{M}_{0}^{*}$ we have

$$
\operatorname{im}(u) \subset W_{N_{0}} \cup E^{+} .
$$

Proof We denote the convex boundary of $W_{n} \subset \mathcal{W}$ by $M^{+}$and the concave boundary by $M_{n}^{-}$. Recall that $M^{+}$is the same for all $W_{n}$. Then we claim that there exists a positive constant $c_{0}>0$ such that all $u \in \mathcal{M}_{0}^{*}$ with $\operatorname{im}(u) \cap M^{+}$and $\operatorname{im}(u) \cap M_{n}^{-}$ both nonempty have energy

$$
E(u) \geq c_{0} n .
$$

This follows from the monotonicity lemma (see Lemma 5.4 below) and the fact that the almost complex structure is periodic. Indeed, we fix a copy of $W_{1}$ in $W_{n}$ and denote for the moment its convex and concave boundary by $\partial W^{+}$and $\partial W^{-}$respectively. We claim that there exists $\tilde{c}>0$ such that any holomorphic curve $v$ with $v^{-1}\left(\partial W^{+}\right) \neq \varnothing$ and $v^{-1}\left(\partial W^{-}\right) \neq \varnothing$ has at least energy $E(v) \geq \tilde{c}$. To see this we observe that each such $v$ has to pass through a point in $W$ with distance $\epsilon_{0}>0$ to the boundary $\partial W^{+} \cup \partial W^{-}$of $W$. Thus we conclude from Lemma 5.4 that $E(v) \geq C \epsilon_{0}^{2}$ for each $v$, where $C$ and $\epsilon_{0}$ only depend on the almost complex structure $J$. Since $J$ is periodic, and a map $u \in \mathcal{M}_{0}^{*}$ with $\operatorname{im}(u) \cap M^{+} \neq \varnothing$ and $\operatorname{im}(u) \cap M_{n}^{-} \neq \varnothing$ passes through the boundaries of $n$ copies of $W_{1}$, Equation (5-3) follows. Using the uniform energy bound for $u \in \mathcal{M}_{0}^{*}$, this implies the proposition in the case where $u_{0}$ has punctures, as every $u \in \mathcal{M}_{0}^{*}$ is then either confined to $E^{+}$or passes through $M^{+}$.

A small modification is required for the case without punctures: here $u_{0} \in \mathcal{M}_{0}^{*}$ is a sphere, and we can choose its lift from $W$ to $\mathcal{W}^{\infty}$ so that without loss of generality, the image of $u_{0}$ intersects $W_{1}$ (ie the first copy). Then we claim that every $u \in \mathcal{M}_{0}^{*}$ intersects $W_{1}$. Otherwise, the fact that $\mathcal{M}_{0}^{*}$ is connected implies the existence of some holomorphic sphere in $\mathcal{M}_{0}^{*}$ that touches $M_{1}^{-}$tangentially from inside $W_{2} \backslash W_{1}$, and this is impossible by convexity. We conclude that every $u \in \mathcal{M}_{0}^{*}$ which escapes from $W_{1} \cup E^{+}$must also pass through $M_{1}^{-}$, so the above argument goes through by using $M_{1}^{-}$in place of $M^{+}$.

For the sake of completeness, we include here the monotonicity lemma; see Hummel [23] for a proof. 
Lemma 5.4 For any compact almost complex manifold $(W, J)$ with Hermitian metric $g$, there are constants $\epsilon_{0}$ and $C>0$ such that the following holds. Assume $(S, j)$ is a compact Riemann surface, possibly with boundary, and $u: S \rightarrow W$ is a pseudoholomorphic curve. Then for every $z \in \operatorname{Int}(S)$ and $r \in\left(0, \epsilon_{0}\right)$ such that $u(\partial S) \cap B_{r}(u(z))=\varnothing$, the following inequality holds:

$$
\text { Area }\left(u(S) \cap B_{r}(u(z))\right) \geq C r^{2}
$$

Since $W_{N_{0}}$ is compact, Proposition 5.3 allows us to apply Corollary 4.9. But this implies that $\mathcal{W}$ is compact, and is thus a contradiction, concluding the proof of Theorems 1.15 and 2.7 .

\subsection{Proof of Theorem 2.8}

Theorem 2.8 follows immediately from Theorem 2.7 and Example 1.3, since a symplectic semifilling with disconnected boundary can always be turned into a closed symplectic 4-manifold containing nonseparating contact hypersurfaces. One can nonetheless give a slightly easier proof as follows.

Assume that the boundary $\partial W$ is disconnected and contains a component $M$ satisfying Property $(\star)$. Thus $W$ satisfies Assumption 4.1, and after attaching cylindrical ends, we obtain a moduli space $\mathcal{M}_{0}^{\mathbf{c}}$ of $J$-holomorphic curves that fill the enlarged manifold $W^{\infty}$. Moreover, all $J$-holomorphic curves have positive punctures going to the end corresponding to $M$. Since they fill $W^{\infty}$, some of these curves must therefore touch $\partial W \backslash M$ tangentially, which is impossible if $\partial W$ is convex.

\subsection{Proof of Theorem 2.9}

Let $(W, \omega)$ be a compact connected 4-manifold with convex boundary $(M, \xi)$ satisfying the Weak Property $(\star)$. After attaching a symplectic cobordism to $\partial W$, we may without loss of generality remove the word "weak". Now assume that $H \subset W \backslash M$ is a nonseparating contact hypersurface. Thus we can cut $W$ open along $H$ and compactify to obtain a connected symplectic cobordism $W_{1}$ with two convex boundary components $\mathrm{H}^{+}$and $\mathrm{M}$, and one concave boundary component $\mathrm{H}^{-}$.

Now we can repeat the construction in the proof of Theorems 1.15 and 2.7, namely we glue infinitely many copies of $W_{1}$ along $H$, obtaining a noncompact symplectic manifold $\mathcal{W}$ with one convex boundary component $H$ and infinitely many convex boundary components which are copies of $M$. From here, we proceed exactly as in the previous proofs, using the moduli space of holomorphic curves arising from Property $(\star)$ on the first copy of $M$. The only new feature is that $\partial \mathcal{W}$ is not compact, 
but since it consists of copies of the same compact and convex components, the results of Section 4 still hold, as convexity prevents the holomorphic curves in $\mathcal{M}_{0}^{\mathbf{c}}$ from ever approaching the other copies of $M$. In particular, Corollary 4.9 applies and again yields a contradiction.

\section{Appendix A Relative nondegeneracy of contact forms}

Our main argument uses holomorphic curves asymptotic to Morse-Bott families of periodic orbits. We prefer not to assume from the start that the contact form is globally Morse-Bott. Thus, we need a perturbation result that preserves a given Morse-Bott submanifold and makes $\lambda$ nondegenerate everywhere else. For this, it suffices to show that one can perturb $\lambda$ in some precompact subset to make all orbits that pass through that subset nondegenerate.

Theorem A.1 Suppose $M$ is a $(2 n-1)$-dimensional manifold with a smooth contact form $\lambda$, and $\mathcal{U} \subset M$ is an open subset with compact closure. Then there exists a Baire subset

$$
\Lambda_{\text {reg }}(\mathcal{U}) \subset\left\{f \in C^{\infty}(M) \mid f>0 \text { and }\left.f\right|_{M \backslash \mathcal{U}} \equiv 1\right\}
$$

such that for each $f \in \Lambda_{\text {reg }}(\mathcal{U})$, every periodic orbit of $X_{f \lambda}$ passing through $\mathcal{U}$ is nondegenerate.

Proof We give a proof in two steps, first showing that a generic choice of the function $f$ makes all simply covered orbits of $X_{f \lambda}$ passing through $\mathcal{U}$ nondegenerate. Then we extend this to multiple covers by a further perturbation.

The first step is an adaptation of the standard Sard-Smale argument. Let $\xi=\operatorname{ker} \lambda$, and for some large $k \in \mathbb{N}$, define the Banach space

$$
C_{\mathcal{U}}^{k}(M)=\left\{f \in C^{k}(M, \mathbb{R})|f|_{M \backslash \mathcal{U}} \equiv 0\right\}
$$

and Banach manifold

$$
\Lambda^{k}(\mathcal{U})=\left\{f \in C^{k}(M, \mathbb{R}) \mid f>0 \text { and } f-1 \in C_{\mathcal{U}}^{k}(M)\right\},
$$

whose tangent space at any $f \in \Lambda^{k}(\mathcal{U})$ can be identified with $C_{\mathcal{U}}^{k}(M)$. We will consider the nonlinear operator

$$
\sigma(x, T, f):=\dot{x}-T X_{f \lambda}(x)
$$

as a section of a Banach space bundle over $H^{1}\left(S^{1}, M\right) \times(0, \infty) \times \Lambda^{k}(\mathcal{U})$ whose fiber at $(x, T, f)$ is $L^{2}\left(x^{*} T M\right)$. Since $X_{f \lambda}$ depends on the first derivative of $f$, it is of class $C^{k-1}$ and the section $\sigma$ is therefore of class $C^{k-2}$. Choosing any symmetric 
connection $\nabla$ on $M$, the linearization of $\sigma$ at $(x, T, f) \in \sigma^{-1}(0)$ with respect to the first variable defines the operator

$$
D_{x}: H^{1}\left(x^{*} T M\right) \rightarrow L^{2}\left(x^{*} T M\right): \hat{x} \mapsto \nabla_{t} \hat{x}-T \nabla_{\hat{x}} X_{f \lambda} .
$$

Since $\dot{x}=T X_{f \lambda}(x)$, we can identify the normal bundle of $x$ with $x^{*} \xi$ and thus define a splitting $x^{*} T M=T S^{1} \oplus x^{*} \xi$. A short calculation then allows us to rewrite $D_{x}$ with respect to the splitting in the block form

$$
D_{x}=\left(\begin{array}{cc}
\partial_{t} & 0 \\
0 & D_{x}^{N}
\end{array}\right)
$$

where $D_{x}^{N}: H^{1}\left(x^{*} \xi\right) \rightarrow L^{2}\left(x^{*} \xi\right)$ is defined again by (A-1), and is a Fredholm operator of index 0 . The orbit $x$ is nondegenerate if and only if $D_{x}^{N}$ is an isomorphism.

The total linearization of $\sigma$ at $(x, T, f) \in \sigma^{-1}(0)$ is now

$$
D \sigma(x, T, f)(\hat{x}, \widehat{T}, \widehat{f})=D_{x} \hat{x}-\widehat{T} X_{f \lambda}(x)-T \hat{X}(x),
$$

where we define the vector field

$$
\widehat{X}:=\left.\partial_{\tau} X_{(f+\tau \widehat{f}) \lambda}\right|_{\tau=0} .
$$

By definition of the Reeb vector field, $\hat{X}$ takes the form $-\widehat{f} X_{f} \lambda+V \hat{f}$ where $V \hat{f} \in \Gamma(\xi)$ is uniquely determined by the condition

$$
\left.d(f \lambda)(V \widehat{f}, \cdot)\right|_{\xi}=\left.d \hat{f}\right|_{\xi}
$$

We define the universal moduli space of parametrized Reeb orbits as $\mathcal{M}:=\sigma^{-1}(0)$, and let $\mathcal{M}^{*} \subset \mathcal{M}$ denote the open subset consisting of triples $(x, T, f)$ for which $x$ is simply covered and $x\left(S^{1}\right) \cap \mathcal{U} \neq \varnothing$. Similarly, denote

$$
\mathcal{M}^{*}(f)=\left\{(x, T) \mid(x, T, f) \in \mathcal{M}^{*}\right\} .
$$

We claim that $D \sigma(x, T, f)$ is surjective whenever $(x, T, f) \in \mathcal{M}^{*}$, hence $\mathcal{M}^{*}$ is a $C^{k-2}$-smooth Banach manifold. To see this, note that one can always find $\eta \in H^{1}\left(T S^{1}\right)$ and $\widehat{T} \in \mathbb{R}$ so that $T x\left(\partial_{t} \eta\right)-\widehat{T} X_{f \lambda}(x)$ takes any desired value in $L^{2}\left(x^{*}\left(\mathbb{R} X_{f \lambda}\right)\right)$, thus it suffices to show that the "normal part"

$$
H^{1}\left(x^{*} \xi\right) \oplus C_{\mathcal{U}}^{k}(M) \rightarrow L^{2}\left(x^{*} \xi\right):(\widehat{x}, \widehat{f}) \mapsto D_{x}^{N} \hat{x}-T V \widehat{f}
$$

is surjective. If it isn't, then there exists a section $\eta \neq 0 \in L^{2}\left(x^{*} \xi\right)$ such that $\left\langle D_{x}^{N} \hat{x}, \eta\right\rangle_{L^{2}}=0$ for all $\hat{x} \in H^{1}\left(x^{*} \xi\right)$ and $\langle V \hat{f}, \eta\rangle_{L^{2}}=0$ for all $\hat{f} \in C_{\mathcal{U}}^{k}(M)$ vanishing outside of $\mathcal{U}$. The first relation implies that $\eta$ is in the kernel of the formal adjoint of $D_{x}^{N}$, a first order linear differential operator, hence $\eta$ is smooth and nowhere vanishing. 
But then if $x\left(t_{0}\right) \in \mathcal{U}$, then using (A-3), $\widehat{f}$ can be chosen near $x\left(t_{0}\right)$ so that the second relation requires $\eta$ to vanish on a neighborhood of $t_{0}$, giving a contradiction.

Now applying the Sard-Smale theorem to the natural projection

$$
\mathcal{M}^{*} \rightarrow \Lambda^{k}(\mathcal{U}):(x, T, f) \mapsto f,
$$

we find a Baire subset $\Lambda_{\text {reg }}^{k}(\mathcal{U}) \subset \Lambda^{k}(\mathcal{U})$ for which every simply covered Reeb orbit passing through $\mathcal{U}$ is nondegenerate.

For the second step, denote by $\operatorname{dist}($,$) the distance functions resulting from any choice$ of Riemannian metrics on $S^{1}$ and $M$, and define for each positive integer $N \in \mathbb{N}$ a subset

$$
\mathcal{M}_{N}(f) \subset \mathcal{M}^{*}(f)
$$

consisting of Reeb orbits $(x, T)$ that satisfy the following conditions:

(1) $T \leq N$.

(2) There exists $t \in S^{1}$ such that

$$
\inf _{t^{\prime} \in S^{1} \backslash\{t\}} \frac{\operatorname{dist}\left(x(t), x\left(t^{\prime}\right)\right)}{\operatorname{dist}\left(t, t^{\prime}\right)} \geq \frac{1}{N} .
$$

(3) There exists $t \in S^{1}$ such that $\operatorname{dist}(x(t), M \backslash \mathcal{U}) \geq 1 / N$.

Moreover, let $\Lambda_{\text {reg, } N}(\mathcal{U}) \subset \Lambda^{\infty}(\mathcal{U})$ denote the space of all smooth functions $f \in \Lambda^{k}(\mathcal{U})$ for which all covers of orbits in $\mathcal{M}_{N}(f)$ up to multiplicity $N$ are nondegenerate. Since nondegeneracy is an open condition and any sequence $\left(x_{k}, T_{k}\right) \in \mathcal{M}_{N}\left(f_{k}\right)$ with $f_{k} \rightarrow f$ in $C^{\infty}$ has a convergent subsequence by the Arzelà-Ascoli theorem, $\Lambda_{\text {reg, } N}(\mathcal{U})$ is an open set. We claim it is also dense. Indeed, any $f \in \Lambda^{\infty}(\mathcal{U})$ has a perturbation $f_{\epsilon} \in \Lambda^{k}(\mathcal{U})$ for which all the simple orbits in $\mathcal{M}_{N}\left(f_{\epsilon}\right)$ are nondegenerate due to step 1 . In this case $\mathcal{M}_{N}\left(f_{\epsilon}\right)$ is a smooth compact 1-manifold, ie a finite union of circles, which are the parametrizations of finitely many distinct nondegenerate orbits, and the space is stable under small perturbations of $f_{\epsilon}$. Thus by a further perturbation, we can make $f_{\epsilon}$ smooth and arrange that none of the orbits in $\mathcal{M}_{N}\left(f_{\epsilon}\right)$ have a Floquet multiplier that is a $k$-th root of unity for $k \in\{1, \ldots, N\}$. The latter can be achieved using a normal form for $f_{\epsilon} \lambda$ as in [21, Lemma 2.3] near each individual orbit: in particular, we can perturb so that each orbit remains unchanged but the linearized return map changes arbitrarily within the space of symplectic linear maps. This proves that $\Lambda_{\text {reg, } N}(\mathcal{U})$ is dense in $\Lambda^{\infty}(\mathcal{U})$, and we can now construct $\Lambda_{\text {reg }}(\mathcal{U})$ as a countable intersection of open dense sets:

$$
\Lambda_{\text {reg }}(\mathcal{U})=\bigcap_{N \in \mathbb{N}} \Lambda_{\text {reg, } N}(\mathcal{U})
$$


Acknowledgments We would like to thank Klaus Mohnke for bringing the question of nonseparating contact hypersurfaces to our attention, John Etnyre for providing Example 1.3, Klaus Niederkrüger, Paolo Ghiggini and Slava Matveyev for enlightening discussions and Yasha Eliashberg for some helpful comments on an earlier draft of the paper. We began work on this article while BB was visiting ETH Zürich, and we'd like to thank ETH for its stimulating working environment. PA was partially supported by NSF grant DMS-0805085. CW was partially supported by NSF Postdoctoral Fellowship DMS-0603500.

\section{References}

[1] C Abbas, Holomorphic open book decompositions arXiv:0907.3512

[2] M Bhupal, K Ono, Symplectic fillings of links of quotient surface singularities arXiv: 0808.3794

[3] F Bourgeois, Y Eliashberg, H Hofer, K Wysocki, E Zehnder, Compactness results in symplectic field theory, Geom. Topol. 7 (2003) 799-888 MR2026549

[4] Y Eliashberg, Filling by holomorphic discs and its applications, from: "Geometry of low-dimensional manifolds, 2 (Durham, 1989)", (S K Donaldson, C B Thomas, editors), London Math. Soc. Lecture Note Ser. 151, Cambridge Univ. Press (1990) 45-67 MR1171908

[5] Y Eliashberg, A few remarks about symplectic filling, Geom. Topol. 8 (2004) 277-293 MR2023279

[6] J B Etnyre, Planar open book decompositions and contact structures, Int. Math. Res. Not. (2004) 4255-4267 MR2126827

[7] J B Etnyre, private communication (2008)

[8] J B Etnyre, K Honda, On symplectic cobordisms, Math. Ann. 323 (2002) 31-39 MR1906906

[9] J B Etnyre, J Van Horn-Morris, Fibered transverse knots and the Bennequin bound arXiv:0803.0758

[10] D T Gay, Four-dimensional symplectic cobordisms containing three-handles, Geom. Topol. 10 (2006) 1749-1759 MR2284049

[11] H Geiges, Examples of symplectic 4-manifolds with disconnected boundary of contact type, Bull. London Math. Soc. 27 (1995) 278-280 MR1328705

[12] H Geiges, Constructions of contact manifolds, Math. Proc. Cambridge Philos. Soc. 121 (1997) 455-464 MR1434654

[13] H Geiges, An introduction to contact topology, Cambridge Studies in Advanced Math. 109, Cambridge Univ. Press (2008) MR2397738 
[14] E Giroux, Links and contact structures, Lecture notes, Georgia Topology Conference (2001) Available at http://www.math.uga.edu/ topology/2001/giroux.pdf

[15] M Gromov, Pseudoholomorphic curves in symplectic manifolds, Invent. Math. 82 (1985) 307-347 MR809718

[16] M Gromov, Partial differential relations, Ergebnisse der Math. und ihrer Grenzgebiete (3) 9, Springer, Berlin (1986) MR864505

[17] H Hofer, Pseudoholomorphic curves in symplectizations with applications to the Weinstein conjecture in dimension three, Invent. Math. 114 (1993) 515-563 MR1244912

[18] H Hofer, V Lizan, J-C Sikorav, On genericity for holomorphic curves in fourdimensional almost-complex manifolds, J. Geom. Anal. 7 (1997) 149-159 MR1630789

[19] H Hofer, K Wysocki, E Zehnder, Properties of pseudo-holomorphic curves in symplectisations. II. Embedding controls and algebraic invariants, Geom. Funct. Anal. 5 (1995) 270-328 MR1334869

[20] H Hofer, K Wysocki, E Zehnder, Properties of pseudoholomorphic curves in symplectisation. IV. Asymptotics with degeneracies, from: "Contact and symplectic geometry (Cambridge, 1994)", (C B Thomas, editor), Publ. Newton Inst. 8, Cambridge Univ. Press (1996) 78-117 MR1432460

[21] H Hofer, K Wysocki, E Zehnder, Properties of pseudoholomorphic curves in symplectisations. I. Asymptotics, Ann. Inst. H. Poincaré Anal. Non Linéaire 13 (1996) 337-379 MR1395676

[22] H Hofer, K Wysocki, E Zehnder, Properties of pseudoholomorphic curves in symplectizations. III. Fredholm theory, from: "Topics in nonlinear analysis", (J Escher, G Simonett, editors), Progr. Nonlinear Differential Equations Appl. 35, Birkhäuser, Basel (1999) 381-475 MR1725579

[23] C Hummel, Gromov's compactness theorem for pseudo-holomorphic curves, Progress in Math. 151, Birkhäuser Verlag, Basel (1997) MR1451624

[24] Y Kanda, The classification of tight contact structures on the 3-torus, Comm. Anal. Geom. 5 (1997) 413-438 MR1487723

[25] R Lutz, Structures de contact sur les fibrés principaux en cercles de dimension trois, Ann. Inst. Fourier (Grenoble) 27 (1977) ix, 1-15 MR0478180

[26] D McDuff, The structure of rational and ruled symplectic 4-manifolds, J. Amer. Math. Soc. 3 (1990) 679-712 MR1049697

[27] D McDuff, Symplectic manifolds with contact type boundaries, Invent. Math. 103 (1991) 651-671 MR1091622

[28] D McDuff, D Salamon, $J$-holomorphic curves and symplectic topology, Amer. Math. Soc. Coll. Publ. 52, Amer. Math. Soc. (2004) MR2045629

[29] E Mora, Pseudoholomorphic cylinders in symplectisations, PhD thesis, New York University (2003) 
[30] H Ohta, K Ono, Simple singularities and symplectic fillings, J. Differential Geom. 69 (2005) 1-42 MR2169581

[31] R Siefring, Intersection theory of punctured pseudoholomorphic curves arXiv: 0907.0470

[32] R Siefring, C Wendl, Pseudoholomorphic curves, intersections and Morse-Bott asymptotics, in preparation

[33] J-Y Welschinger, Effective classes and Lagrangian tori in symplectic four-manifolds, J. Symplectic Geom. 5 (2007) 9-18 MR2371182

[34] C Wendl, Automatic transversality and orbifolds of punctured holomorphic curves in dimension four, to appear in Comment. Math. Helv. arXiv:0802.3842

[35] C Wendl, Compactness for embedded pseudoholomorphic curves in 3-manifolds, to appear in J. Eur. Math. Soc. (JEMS) arXiv:math/0703509

[36] C Wendl, Contact fiber sums, monodromy maps and symplectic fillings, in preparation

[37] C Wendl, Holomorphic curves in blown up open books arXiv:1001.4109

[38] C Wendl, Open book decompositions and stable Hamiltonian structures, to appear in Expos. Math. arXiv:0808.3220

[39] C Wendl, Punctured holomorphic curves with boundary in 3-manifolds: Fredholm theory and embededdness, in preparation

[40] C Wendl, Strongly fillable contact manifolds and J-holomorphic foliations, to appear in Duke Math. J. arXiv:0806.3193

[41] C Wendl, Finite energy foliations and surgery on transverse links, $\mathrm{PhD}$ thesis, New York University (2005)

[42] K Zehmisch, The Eliashberg-Gromov tightness theorem, Diplom Thesis, Universität Leipzig (2003)

Department of Mathematics, Purdue University

150 N University Street, West Lafayette, IN 47907-2067, USA

Max-Planck Institut

Inselstrasse-22, 04103 Leipzig, Germany

Institut für Mathematik, Humboldt-Universität zu Berlin

10099 Berlin, Germany

palbers@math.purdue.edu, bramham@mis.mpg.de, wendl@mathematik.hu-berlin.de

Received: 22 July 2009 